\title{
Prediction of Co and Ru nanocluster morphology on 2D MoS 2 from interaction energies
}

\author{
Cara-Lena Nies ${ }^{1}$ and Michael Nolan ${ }^{*} 1,2$
}

\section{Full Research Paper}

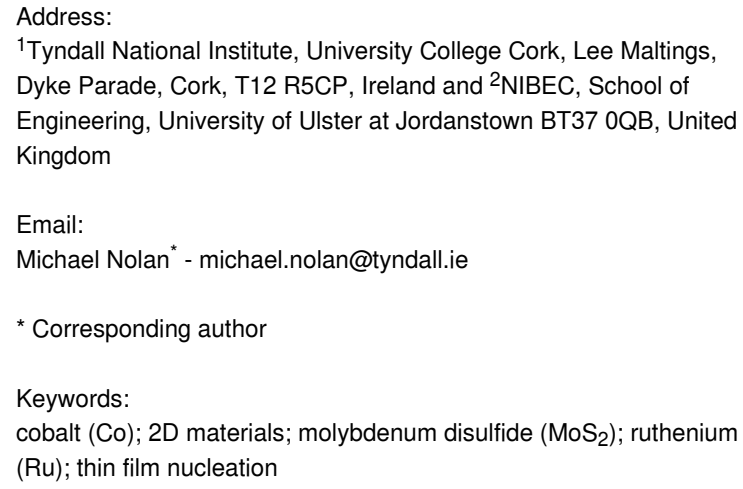

\author{
Beilstein J. Nanotechnol. 2021, 12, 704-724. \\ https://doi.org/10.3762/bjnano.12.56 \\ Received: 18 March 2021 \\ Accepted: 29 June 2021 \\ Published: 14 July 2021 \\ Associate Editor: E. Meyer \\ (C) 2021 Nies and Nolan; licensee Beilstein-Institut. \\ License and terms: see end of document.
}

\begin{abstract}
Layered materials, such as $\mathrm{MoS}_{2}$, have a wide range of potential applications due to the properties of a single layer, which often differ from the bulk material. They are of particular interest as ultrathin diffusion barriers in semiconductor device interconnects and as supports for low-dimensional metal catalysts. Understanding the interaction between metals and the $\mathrm{MoS}_{2}$ monolayer is of great importance when selecting systems for specific applications. In previous studies the focus has been largely on the strength of the interaction between a single atom or a nanoparticle of a range of metals, which has created a significant knowledge gap in understanding thin film nucleation on 2D materials. In this paper, we present a density functional theory (DFT) study of the adsorption of small Co and Ru structures, with up to four atoms, on a monolayer of $\mathrm{MoS}_{2}$. We explore how the metal-substrate and metal-metal interactions contribute to the stability of metal clusters on $\mathrm{MoS}_{2}$, and how these interactions change in the presence of a sulfur vacancy, to develop insight to allow for a prediction of thin film morphology. The strength of interaction between the metals and $\mathrm{MoS}_{2}$ is in the order $\mathrm{Co}>\mathrm{Ru}$. The competition between metal-substrate and metal-metal interaction allows us to conclude that 2D structures should be preferred for Co on $\mathrm{MoS}_{2}$, while Ru prefers 3D structures on $\mathrm{MoS}_{2}$. However, the presence of a sulfur vacancy decreases the metal-metal interaction, indicating that with controlled surface modification 2D Ru structures could be achieved. Based on this understanding, we propose $\mathrm{Co}$ on $\mathrm{MoS}_{2}$ as a suitable candidate for advanced interconnects, while Ru on $\mathrm{MoS}_{2}$ is more suited to catalysis applications.
\end{abstract}

\section{Introduction}

Layered materials that can be exfoliated into 2D sheets continue to generate significant interest across various disciplines, including batteries [1,2], catalysis [3,4], electronics [5-10], photonics [11,12], and sensors [13-16]. This is due in part to the interesting properties of these 2D materials, which often differ from their bulk equivalent, as well as the flexibility in fabrica- 
tion afforded by an ultrathin material [17]. The majority of applications are built on an interaction between a metal and the 2D material. There are multiple studies in this regard that involve the adsorption of or doping with transition metals [4,5,18-22], alkali and alkali earth metals [23-25], and nonmetals [25] on $\mathrm{MoS}_{2}$ and other 2D materials. While experimental studies can be used to probe the performance of the 2D material in a device or some of the interfacial interactions between metal and 2D materials $[9,10,21,26]$, first principles modelling is a powerful tool that permits the investigation of the detailed interactions of metals and 2D materials at the atomic scale. In particular, understanding the nucleation of metals on $2 \mathrm{D}$ materials will be valuable for the design of catalysts or for preventing islanding of conductive metals.

Typically, theoretical studies focus on the adsorption of either single atoms of a series of metals [21,23-25,27] or large nanoparticle-like structures $[19,20]$. In our previous study we identified that while these studies do deliver useful insights, there is a knowledge gap in the understanding of metal thin film nucleation on 2D materials [28]. We showed that we can investigate the first stages of thin film nucleation on 2D materials with first principles simulations, using the example of small $\mathrm{Cu}_{n}$ structures on an $\mathrm{MoS}_{2}$ monolayer (ML).

$\mathrm{MoS}_{2}$ is a naturally occurring transition metal dichalcogenide (TMD) and one of the most frequently studied 2D materials Unlike graphene, $\mathrm{MoS}_{2}$ is a semiconductor, which gives it an increased number of possible applications [11,29]. Our previous first principles study [28] of the interaction of $\mathrm{Cu}$ species on $\mathrm{MoS}_{2}$ showed how $\mathrm{Cu}$ can take different structures depending on the number of $\mathrm{Cu}$ atoms and whether the TMD is stoichiometric or defective.

In the present study, we will expand the knowledge gained from our previous work on $\mathrm{Cu}$ on $\mathrm{MoS}_{2}$ and apply it to the adsorption of small $\mathrm{Co}_{n}$ and $\mathrm{Ru}_{n}$ clusters on an $\mathrm{MoS}_{2} \mathrm{ML}$, where $n=$ 1-4. Co and Ru are of great interest in conjunction with $\mathrm{MoS}_{2}$ for application in advanced interconnects as alternatives to $\mathrm{Cu}$ [30-35] and TaN. Applications in catalysis include Pt-free hydrogen evolution catalysts [36-41].

Interconnects require high-quality, conformal thin films with low resistivity, to avoid many of the typical failure mechanisms such as electromigration $[42,43]$. This means that $3 \mathrm{D}$ migration of atoms (agglomeration) should be inhibited, while 2D growth (wetting) should be promoted. In contrast, in catalysis applications the ratio of surface to bulk is of great importance in promoting catalytic activity. Therefore, 3D growth (agglomeration) is essential when creating a supported metal catalyst [4447].
In this work we aim to determine the atomic-scale interactions that control the stability of small $\mathrm{Co}_{n}$ and $\mathrm{Ru}_{n}$ clusters $(n=1-4)$ on a single $\mathrm{ML}$ of $\mathrm{MoS}_{2}$. Based on this understanding, alongside the magnitude of metal-substrate and metal-metal interactions we will be able to predict the morphology of Co and $\mathrm{Ru}$ thin films on 2D MoS 2 . We have previously studied 2D and 3D $\mathrm{Cu}$ clusters on $\mathrm{TaN}$, where we determined that there are two useful descriptors for 2D-vs-3D growth [48]: (1) If the metal-substrate interaction is more favourable than the metal-metal interaction, then 2D growth is preferred; and (2) if the total binding energy is more favourable than the cohesive energy of the bulk metal, then $2 \mathrm{D}$ growth is preferred.

Predictions made using these descriptors can be used when deciding which metal-substrate combination will be suitable for a particular application where the shape of the metal is vital.

\section{Methods}

All calculations for this study were carried out with density functional theory (DFT) using the Vienna Ab initio Simulation Package (VASP) version 5.4 [49]. Three-dimensional boundary conditions were applied and the spin-polarized general gradient approximation (GGA) along with the Perdew-Burke-Ernzerhof (PBE) approximation to the exchange-correlation functional were used to describe the system [50]. Valence electrons were described explicitly using a plane-wave basis set with an energy cutoff of $450 \mathrm{eV}$. The valence electron configurations are as follows: $\mathrm{Co}=4 \mathrm{~s}^{2} 3 \mathrm{~d}^{7}, \mathrm{Ru}=5 \mathrm{~s}^{1} 4 \mathrm{~d}^{7}, \mathrm{Mo}=5 \mathrm{~s}^{1} 4 \mathrm{~d}^{5}$, and $\mathrm{S}=3 \mathrm{~s}^{2}$ $3 p^{4}$. The core electrons were treated with the projectoraugmented wave potential (PAW) [51]. A Monkhorst-Pack k-point grid of 221 was used. All forces acting on the atoms were converged to within $0.02 \mathrm{eV} / \AA$. A Methfessel-Paxton smearing of order 1 was used and no symmetry was applied.

The description of pristine and defective $\mathrm{MoS}_{2}$ monolayers (ML) was published in our previous work [28]. Bulk $\mathrm{MoS}_{2}$ is made up of two layers. To create the pristine ML one of these was removed, which also creates the vacuum necessary to avoid interaction along the $z$-axis; the vacuum region is $8 \AA$. $\mathrm{A}(5 \times 5)$ super cell was used. No van der Waals (vdW) corrections were applied, as both the literature and our own tests (Supporting Information File 1, section S4) show that vdW forces do not dominate in these types of structures. The defective ML has the same structure as the pristine ML, except that a single $\mathrm{S}$ atom has been removed to create a vacancy and the ions are relaxed with no symmetry constraints. Using $\mathrm{H}_{2} \mathrm{~S}$ as a reference, we have computed an exothermic vacancy formation energy of $-6.16 \mathrm{eV}$. The bond lengths in bulk structures that are used for comparison are based on the crystal structures in [52-57]. Only a theoretical crystal structure was available for RuMo, all other structures used have been determined experimentally. 
To understand the binding of $\mathrm{Co}$ and $\mathrm{Ru}$ to the $\mathrm{MoS}_{2}$ monolayer, four different energies are computed:

1. Binding energy per metal atom:

$$
E_{\text {bind/atom }}=\frac{\left(E_{\text {total }}-E_{\text {monolayer }}-n E_{\text {metal_atom }}\right)}{n}
$$

$E_{\text {total }}$ is the total energy of the relaxed $\mathrm{Co}_{n}$ or $\mathrm{Ru}_{n}(n=1-4)$ adsorbed on $\mathrm{MoS}_{2}$. The energy of a single gas-phase metal atom $\left(E_{\text {metal_atom }}\right)$ is multiplied by $n$, the number of atoms in adsorbed $\mathrm{Co}_{n}$ or $\mathrm{Ru}_{n}$.

2. Binding energy with reference to a free $\mathrm{Co}_{n}$ or $\mathrm{Ru}_{n}$ cluster:

$$
E_{\text {metal--substrate }}=\frac{E_{\text {total }}-E_{\text {monolayer* }}-E_{\text {metal_cluster }}}{n}
$$

where $E_{\text {metal_cluster }}$ is the single point energy of the $\mathrm{Co}_{n}$ or $\mathrm{Ru}_{n}$ nanocluster structure in vacuum. $E_{\text {monolayer* }}$ is the single point energy of the monolayer after relaxation. We chose to use this instead of the reference used in Equation 1, as surface rearrangements occur in several of the various structures. This method of computing the binding energy isolates the metal-substrate interaction. Using these two methods of computing the binding energy also allows us to determine an approximate metal-metal interaction energy, by applying Equation 3:

3. Metal-metal interaction energy:

$$
E_{\text {interact }}=E_{\text {bind/atom }}-E_{\text {metal-substrate }}
$$

\section{Addition energy:}

$$
E_{\text {add }}=E_{\text {total }}-E_{\text {monolayer }+(\mathrm{n}-1) \text { metal }}-E_{\text {metal_atom }}
$$

where $n$ is the number of $\mathrm{Co}$ or Ru atoms. This models the addition of a metal atom to an existing adsorbed cluster with $(n-1)$ $\mathrm{Co}$ or $\mathrm{Ru}$ atoms.

\section{Results and Discussion Ru and Co on Pristine $\mathrm{MoS}_{2}$}

As in our previous work [28], there are three metal atom adsorption sites, labelled as atop_S, atop_Mo, and hollow, on the $\mathrm{MoS}_{2} \mathrm{ML}$, which are highlighted in Figure 1A. Site atop_S has a metal atom adsorbed directly atop a $\mathrm{S}$ atom. Site atop_Mo has a metal atom binding to three $\mathrm{S}$ atoms directly above a Mo atom and site hollow has a metal atom binding to three $\mathrm{S}$ atoms, but with no Mo atom underneath.
To study how $\mathrm{Ru}$ and $\mathrm{Co}$ atoms begin to nucleate into a film on the $\mathrm{MoS}_{2} \mathrm{ML}$ and to compare with the behaviour of $\mathrm{Cu}$ on $\mathrm{MoS}_{2}$, we adsorb $\mathrm{Co}_{n}$ and $\mathrm{Ru}_{n}$ species with one, two, three, and four atoms on $\mathrm{MoS}_{2}$. The binding energies for the set of adsorption structures calculated using Equation 1 are shown in Table 1. Metal-substrate interaction energies calculated from Equation 2 are shown in Table 2, metal-metal interaction energies calculated from Equation 3 are shown in Table 3, and addition energies calculated with Equation 4 are shown in Table S1 of Supporting Information File 1. A structure is considered 2D when all metal atoms are bound directly to the $\mathrm{MoS}_{2} \mathrm{ML}$. For $3 \mathrm{D}$ structures, at least one of the metal atoms is bound to other metal atoms, but not to $\mathrm{MoS}_{2}$.

The relaxed geometries of the various $\mathrm{Co}_{n}$ species are shown in Figure 1 and below in Figure 3, Figure 5, and Figure 7, while the geometries for $\mathrm{Ru}_{n}$ species are shown below in Figure 2, Figure 4, Figure 6, and Figure 8. The energies shown in these figures are the binding energies computed using Equation 1. Structures are referred to and labelled according to the initial cluster adsorption structure, to avoid confusion due to any geometry rearrangements that occur.

In the following sections we elaborate how factors influencing structure stability vary as the cluster size increases. Such factors include the presence or absence of metal-metal bonds, symmetrical versus asymmetrical addition, incorporation of atoms into the ML, adsorption sites, and changes to stability in the presence of an S vacancy. Our findings are supported through analysis of geometry variations, bond lengths, adsorption, addition and metal-metal interaction energies, and Bader charges.

\section{Single-atom adsorption}

$\mathrm{Co}$ and $\mathrm{Ru}$ atoms adsorb exothermically at all three adsorption sites. A single Co atom adsorbs most strongly at site atop_Mo, with an energy gain of $-5.82 \mathrm{eV}$, followed by site hollow and site atop_S, with energy gains of $-5.55 \mathrm{eV}$ and $-4.21 \mathrm{eV}$, respectively. A single Ru atom adsorbs preferentially at site atop_Mo with an energy gain of $-3.92 \mathrm{eV}$, followed by site hollow and site atop_S, with energy gains of $-3.40 \mathrm{eV}$ and $-2.47 \mathrm{eV}$, respectively.

Similarly to $\mathrm{Cu}$, for both $\mathrm{Co}$ and $\mathrm{Ru}$, site atop_Mo is the most favourable adsorption site. This is likely due to the adatom position repeating the geometry of the $\mathrm{MoS}_{2} \mathrm{ML}$. We find that for a single adatom, $\mathrm{Co}-\mathrm{S}$ distances are the shortest of all the metals studied, lying between 1.99 and $2.16 \AA$ depending on the adsorption site. These Co-S distances are shorter than the Co-S distance of $2.31 \AA$ in bulk $\mathrm{CoS}_{2}$ [53]. Similarly, the Ru-S distances are between 2.13 and $2.24 \AA$, compared to $2.37 \AA$ in bulk $\mathrm{RuS}_{2}$ [56]. The shortest metal-S bonds are measured at site 


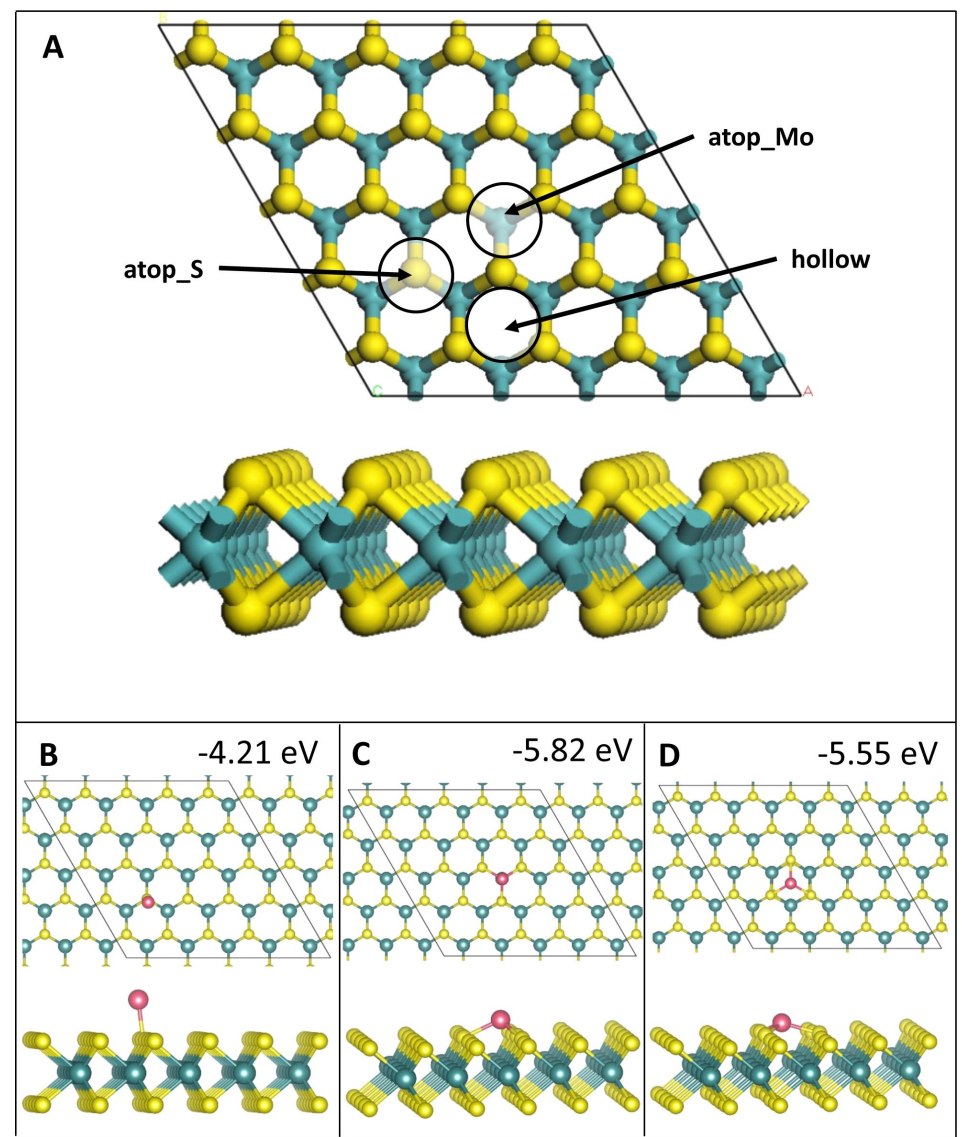

Figure 1: Atomic structure and energies of $\mathrm{Co}_{1}$ adsorption modes on perfect $\mathrm{MoS}_{2}$. Mo is shown in teal, $\mathrm{S}$ in yellow, Co in pink and Ru in purple throughout the article.

\begin{tabular}{|c|c|c|c|c|c|c|c|}
\hline \multirow{2}{*}{$\begin{array}{l}\text { No. of metal } \\
\text { atoms }\end{array}$} & \multirow[t]{2}{*}{ Configuration } & \multicolumn{3}{|c|}{$E_{\text {bind }} /$ Co-atom [eV] } & \multicolumn{3}{|c|}{$E_{\text {bind }} /$ Ru-atom [eV] } \\
\hline & & S_atop & Mo_atop & hollow & S_atop & Mo_atop & hollow \\
\hline 1 & - & -4.21 & -5.82 & -5.55 & -2.47 & -3.92 & -3.40 \\
\hline \multirow[t]{3}{*}{2} & neighbouring & -6.05 & -6.05 & -5.73 & -4.45 & -4.45 & -3.75 \\
\hline & separated & -4.20 & -5.81 & -4.92 & -2.45 & -3.92 & -3.19 \\
\hline & non-equivalent & -5.56 & -5.37 & -5.85 & -3.99 & -3.71 & -3.87 \\
\hline \multirow[t]{3}{*}{3} & line & -5.61 & -6.08 & -5.88 & -4.07 & -4.47 & -4.41 \\
\hline & triangle & -5.43 & -6.13 & -5.89 & -2.57 & -4.65 & -4.46 \\
\hline & 3D triangle & -5.53 & -6.05 & -5.56 & -4.22 & -4.72 & -4.36 \\
\hline \multirow[t]{4}{*}{4} & line & -6.11 & -6.10 & -5.95 & -4.28 & -4.55 & -4.76 \\
\hline & rhombus & -5.86 & -5.93 & -5.87 & -4.61 & -4.28 & -4.37 \\
\hline & 3D rectangle & -6.28 & -6.33 & -5.93 & -3.29 & -4.93 & -4.62 \\
\hline & tetrahedral & -5.85 & -6.32 & -6.05 & -4.70 & -5.14 & -4.74 \\
\hline
\end{tabular}


Table 2: Computed metal-substrate interaction energies for $\mathrm{Co}_{2}, \mathrm{Co}_{3}, \mathrm{Co}_{4}, \mathrm{Ru}_{2}, \mathrm{Ru}_{3}$ and $\mathrm{Ru}_{4}$ on a MoS $\mathrm{ML}$ for various atom configurations using Equation 2. For the "non-equivalent" configurations for two metal atom adsorption, the column "S_atop site" has atoms at sites S_atop and Mo_atop, "Mo_atop site" has atoms at S_atop and hollow, and "hollow site" has atoms at Mo_atop and hollow.

\begin{tabular}{|c|c|c|c|c|c|c|c|}
\hline \multirow{2}{*}{$\begin{array}{l}\text { No. of metal } \\
\text { atoms }\end{array}$} & \multirow[t]{2}{*}{ Configuration } & \multicolumn{3}{|c|}{$E_{\text {metal-substrate }} /$ Co-atom $[\mathrm{eV}]$} & \multicolumn{3}{|c|}{$E_{\text {metal-substrate }} /$ Ru-atom $[\mathrm{eV}]$} \\
\hline & & S_atop & Mo_atop & hollow & S_atop & Mo_atop & hollow \\
\hline \multirow[t]{3}{*}{2} & neighbouring & -2.71 & -2.64 & -2.97 & -2.71 & -2.67 & -4.55 \\
\hline & separated & -1.47 & -3.00 & -2.05 & 0.58 & -1.21 & -0.60 \\
\hline & non-equivalent & -1.44 & -1.54 & -2.02 & -1.61 & -1.36 & -3.96 \\
\hline \multirow[t]{3}{*}{3} & line & -1.17 & -2.78 & -4.78 & -0.79 & -3.17 & -5.14 \\
\hline & triangle & -1.66 & -2.31 & -2.29 & 0.12 & -2.07 & -2.61 \\
\hline & 3D triangle & -0.77 & -1.42 & -0.92 & -0.66 & -1.32 & -1.88 \\
\hline \multirow[t]{4}{*}{4} & line & -1.79 & -2.46 & -3.77 & -0.16 & -14.10 & -4.65 \\
\hline & rhombus & -0.69 & -0.98 & -3.42 & 0.62 & 1.01 & -0.86 \\
\hline & 3D rectangle & -1.69 & -1.55 & -1.50 & -0.24 & -1.24 & -1.49 \\
\hline & tetrahedral & -0.85 & -1.69 & -2.40 & -0.79 & -1.67 & -5.19 \\
\hline
\end{tabular}

Table 3: Computed metal-metal interaction energies using Equation 3. For the "non-equivalent" configurations for two metal atom adsorption, the column "S_atop site" has atoms at sites S_atop and Mo_atop, "Mo_atop site" has atoms at S_atop and hollow, and "hollow site" has atoms at Mo_atop and hollow.

\begin{tabular}{|c|c|c|c|c|c|c|c|}
\hline \multirow{2}{*}{$\begin{array}{l}\text { No. of metal } \\
\text { atoms }\end{array}$} & \multirow[t]{2}{*}{ Configuration } & \multicolumn{3}{|c|}{$E_{\text {interact }} /$ Co-atom $[\mathrm{eV}]$} & \multicolumn{3}{|c|}{$E_{\text {interact }} /$ Ru-atom $[\mathrm{eV}]$} \\
\hline & & S_atop & Mo_atop & hollow & S_atop & Mo_atop & hollow \\
\hline \multirow[t]{3}{*}{2} & neighbouring & -3.34 & -3.42 & -2.76 & -1.74 & -1.77 & 0.79 \\
\hline & separated & -2.73 & -2.82 & -2.88 & -3.04 & -2.71 & -2.59 \\
\hline & non-equivalent & -4.11 & -3.84 & -3.84 & -2.38 & -2.34 & -0.09 \\
\hline \multirow[t]{3}{*}{3} & line & -4.43 & -3.30 & -1.09 & -3.28 & -1.30 & 0.73 \\
\hline & triangle & -0.98 & -3.82 & -3.60 & -2.68 & -2.58 & -1.86 \\
\hline & 3D triangle & -4.77 & -4.63 & -4.64 & -3.56 & -3.40 & -2.48 \\
\hline \multirow[t]{4}{*}{4} & line & -4.32 & -3.64 & -2.18 & -4.12 & 9.56 & -0.11 \\
\hline & rhombus & -5.17 & -4.95 & -2.45 & -5.23 & -5.29 & -3.50 \\
\hline & $3 \mathrm{D}$ rectangle & -4.59 & -4.79 & -4.43 & -3.05 & -3.69 & -3.13 \\
\hline & tetrahedral & -5.00 & -4.63 & -3.66 & -3.92 & -3.48 & -4.72 \\
\hline
\end{tabular}

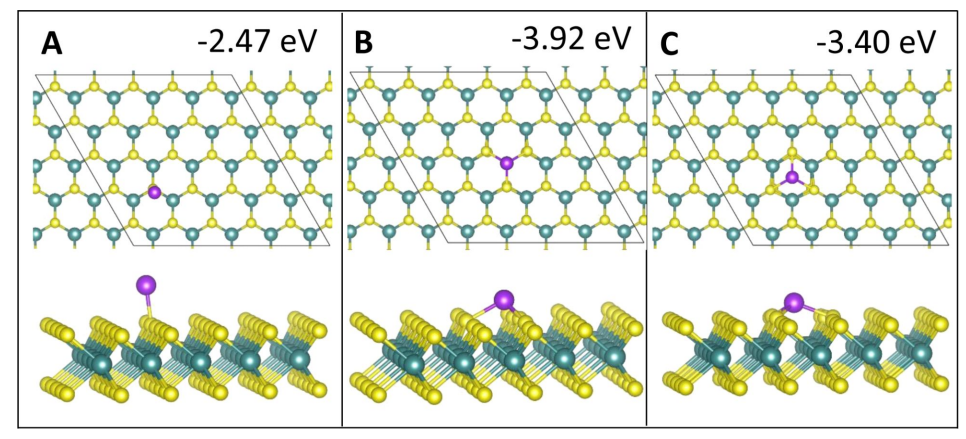

Figure 2: Atomic structure and energies of $\mathrm{Ru}_{1}$ adsorption modes on perfect $\mathrm{MoS}_{2}$. 
atop_S, compared to those measured at sites atop_Mo and hollow. There are some Co-Mo bonds observed, with Co-Mo distances of $2.85 \AA$, but there are no $\mathrm{Ru}-\mathrm{Mo}$ bonds or $\mathrm{Cu}-\mathrm{Mo}$ bonds [28]; the presence of these could be one origin for the enhanced interaction between Co and the $\mathrm{MoS}_{2}$ ML. The relaxed geometries for adsorption of $\mathrm{Co}_{1}$ and $\mathrm{Ru}_{1}$ are shown in Figure 1B-D and Figure 2, respectively.

\section{Two-atom adsorption}

For two-adatom adsorption, there are three different $\mathrm{M}_{2}$ geometries. All of these are 2D. They involve atoms adsorbed at nearest neighbour equivalent surface sites, at equivalent but separated sites and atoms at neighbouring but non-equivalent sites. Relaxed geometries for adsorption of $\mathrm{Co}_{2}$ and $\mathrm{Ru}_{2}$ are shown in Figure 3 and Figure 4, respectively.
For Co, adsorbing two atoms at equivalent neighbour sites corresponding to adsorption site atop_Mo is the most favourable $\mathrm{Co}_{2}$ configuration, with a computed adsorption energy of $-6.05 \mathrm{eV} /$ atom. Adatoms originally adsorbed as equivalent neighbours at site atop_S migrate to site atop_Mo instead (Figure 3A,B). This adsorption mode is more favourable by $0.32 \mathrm{eV}$ compared to equivalent neighbours at site hollow (Figure 3C).

The adsorption of two Co atoms is preferred at equivalent, separated sites corresponding to adsorption mode atop_Mo (Figure $3 \mathrm{E}$ ). We propose that this is due to the geometry match with the substrate as there are no $\mathrm{Co}-\mathrm{Co}$ bonds to contribute to the binding energy, only the weaker adsorption has Co-Mo bonds and both adsorption sites have metal interaction energies

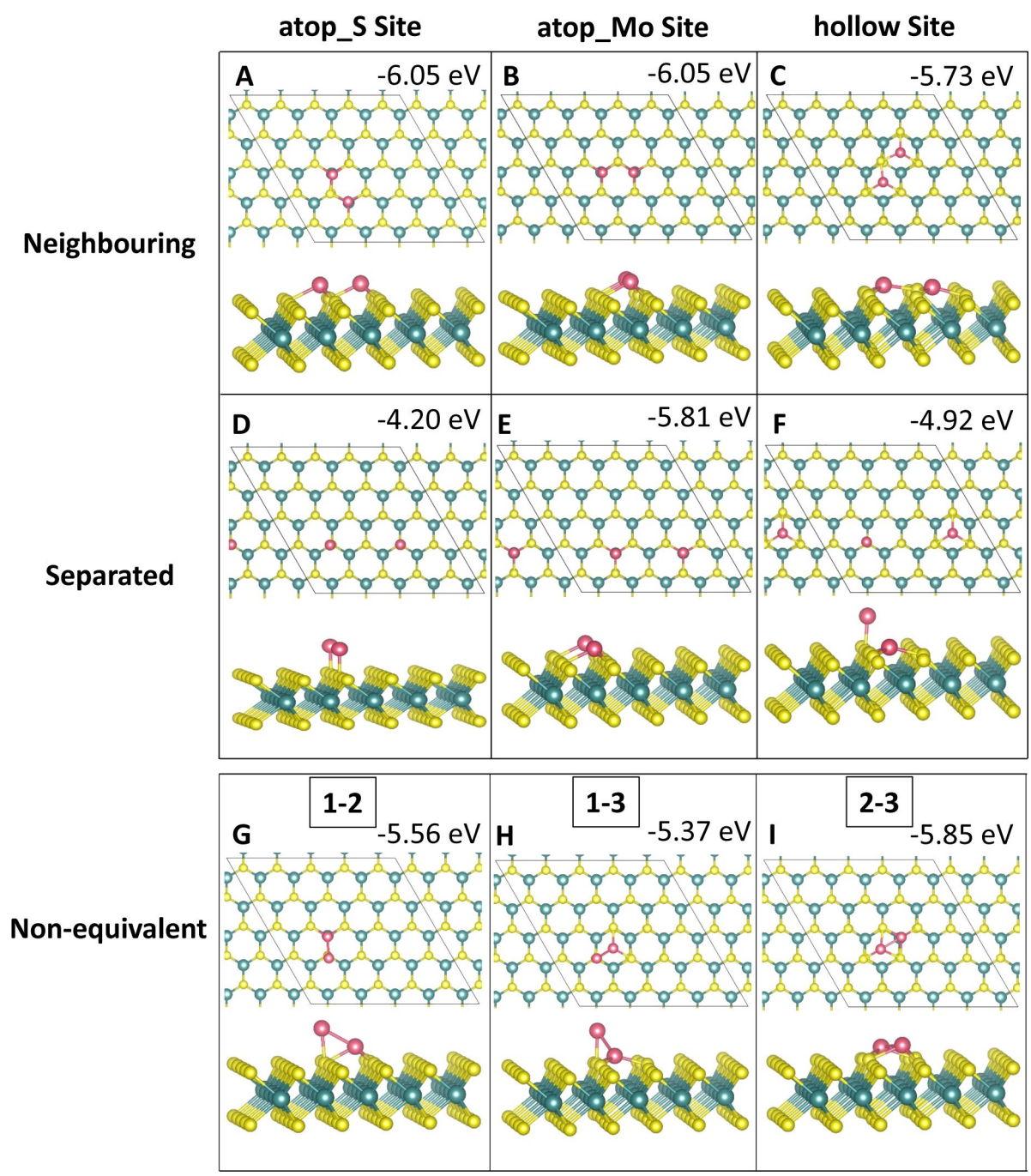

Figure 3: Atomic structure and energies of $\mathrm{Co}_{2}$ adsorption modes on perfect $\mathrm{MoS}_{2}$. 


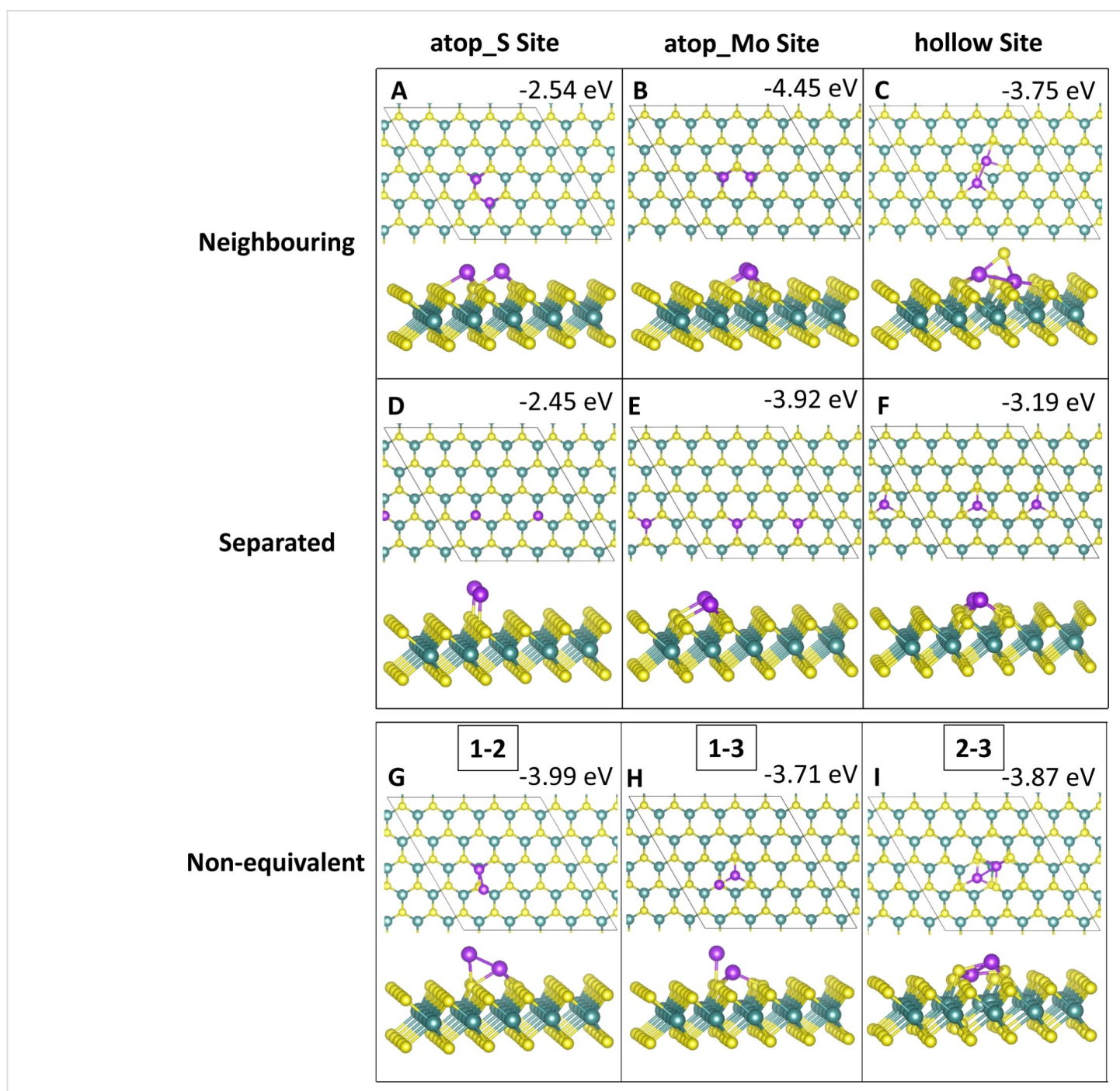

Figure 4: Atomic structure and energies of $\mathrm{Ru}_{2}$ adsorption modes on perfect $\mathrm{MoS}_{2}$

within less than $0.1 \mathrm{eV}$ of each other. Adsorption of separated atoms at site atop_S is the least favourable configuration for $\mathrm{Co}_{2}$ (Figure 3D).

Starting from a configuration of $\mathrm{Co}_{2}$ adsorbed at site hollow, during relaxation one Co atom migrated to site atop_S, making this a non-equivalent adsorption at separated sites atop_S hollow, as shown in Figure 3F. This explains the less favourable binding energy of $-4.92 \mathrm{eV}$ compared to $-5.73 \mathrm{eV}$ at equivalent neighbouring sites hollow and $-5.37 \mathrm{eV}$ at nonequivalent neighbouring sites atop_S - hollow.

Adsorption of Co atoms at neighbouring but non-equivalent sites is overall more favourable than adsorption of separated atoms, in particular where the Co atoms bind to sites atop_Mo hollow (Figure 3I). These structures also have the strongest metal-metal interaction energies (Table 3 ). This is due to
$\mathrm{Co}-\mathrm{Co}$ bonding, which is present exclusively for these $\mathrm{Co}_{2}$ adsorption modes due to the decreased distance between atoms compared to equivalent sites. We also observe large addition energies for these structures, showing that addition of a second atom can stabilise any atoms that may be initially adsorbed at a less favourable site.

During the adsorption of $\mathrm{Ru}_{2}$, several rearrangements were observed. While adsorption of two neighbouring equivalent $\mathrm{Ru}$ atoms at site atop_Mo is most favourable (Figure 4B), with $E_{\text {bind }}=-4.45 \mathrm{eV}$, the initial adsorption at neighbouring equivalent sites atop_S resulted in both atoms migrating to site atop_Mo (Figure 4B), similar to $\mathrm{Co}_{2}$, indicating that this particular configuration is unstable for metal atoms adsorbed at site atop_S. The adsorption of $\mathrm{Ru}$ at separated sites atop_S (Figure 4D) is the least favourable configuration for $\mathrm{Ru}_{2}$, as well, although no rearrangements or migration occur here. 
Similar to $\mathrm{Co}_{2}$, adsorption of $\mathrm{Ru}$ at equivalent separated sites atop_Mo (Figure 4E) is the second most favourable of the equivalent $\mathrm{Ru}_{2}$ adsorptions. The large distortions of the $\mathrm{MoS}_{2}$ $\mathrm{ML}$ for an initial configuration of neighbouring $\mathrm{Ru}$ at site hollow (Figure 4C), are caused by the attempted incorporation of one $\mathrm{Ru}$ atom into the $\mathrm{S}$ layer during relaxation. This has also caused an S atom adjacent to Ru to move out of the surface and bond to both $\mathrm{Ru}$ atoms from above, forming a triangular $\mathrm{Ru}_{2} \mathrm{~S}$ structure. With a binding energy of $-3.99 \mathrm{eV}$, the combination of sites atop_S - atop_Mo (Figure 4G) is as favourable as separated atoms at site atop_Mo (Figure 4E). This indicates, that at this very early stage of film growth there is no preference yet between structures with separated atoms and those with $\mathrm{Ru}-\mathrm{Ru}$ bonds.

Those configurations that underwent strong rearrangement during relaxation, that is, neighbouring equivalent atoms at site hollow (Figure 4C) and neighbouring atoms at the non-equivalent site atop_Mo - hollow (Figure 4I) have very weak metal-metal interaction energies (Table 3 ). The neighbouring site hollow has a positive metal-metal interaction energy, while the non-equivalent site atop_Mo - hollow has an interaction energy close to zero. The latter should mean that metal atoms do not agglomerate or separate, while a positive metal-metal interaction should be indicative of a separation of atoms. However, a zero or positive metal-metal energy can only be com-

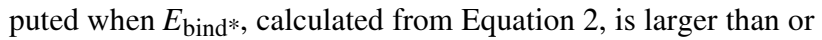
almost equal to $E_{\text {bind }}$, calculated from Equation 1 . As $E_{\text {bind* }}$ is calculated using the structure of the $\mathrm{MoS}_{2} \mathrm{ML}$ after metal relaxation and the energy of the Ru cluster in vacuum as references, this indicates that for these structures $E_{\text {bind }}$ reflects energy changes during rearrangement, which in turn affects the magnitude of the metal-metal interaction energy.

\section{Three-atom adsorption}

Three-atom adsorption involved the study of three different geometries, two of which are $2 \mathrm{D}$ and one of which is $3 \mathrm{D}$. These are shown in Figure 5 and Figure 6. For the first configuration,

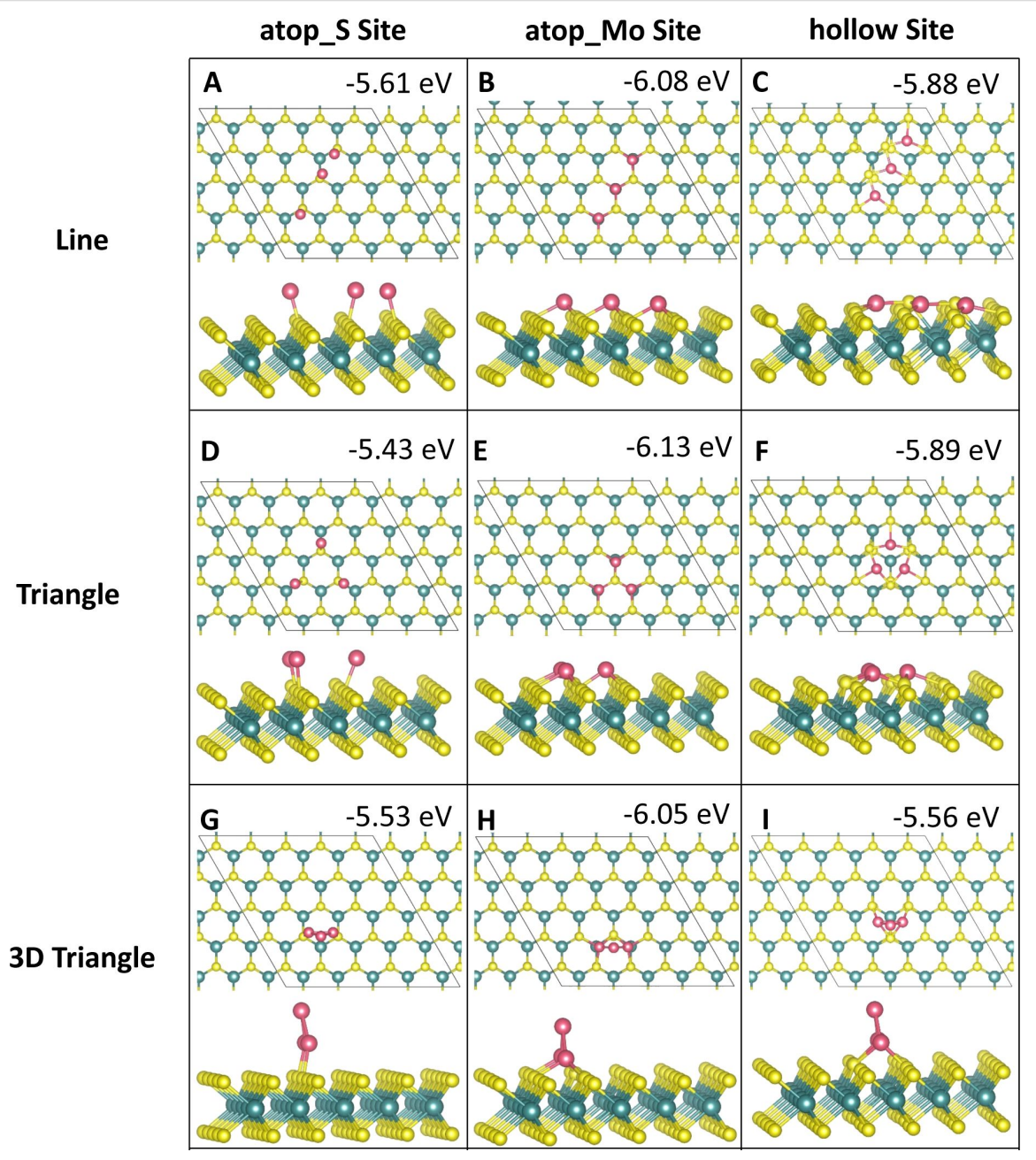

Figure 5: Atomic structure and energies of $\mathrm{Co}_{3}$ adsorption modes on perfect $\mathrm{MoS}_{2}$. 


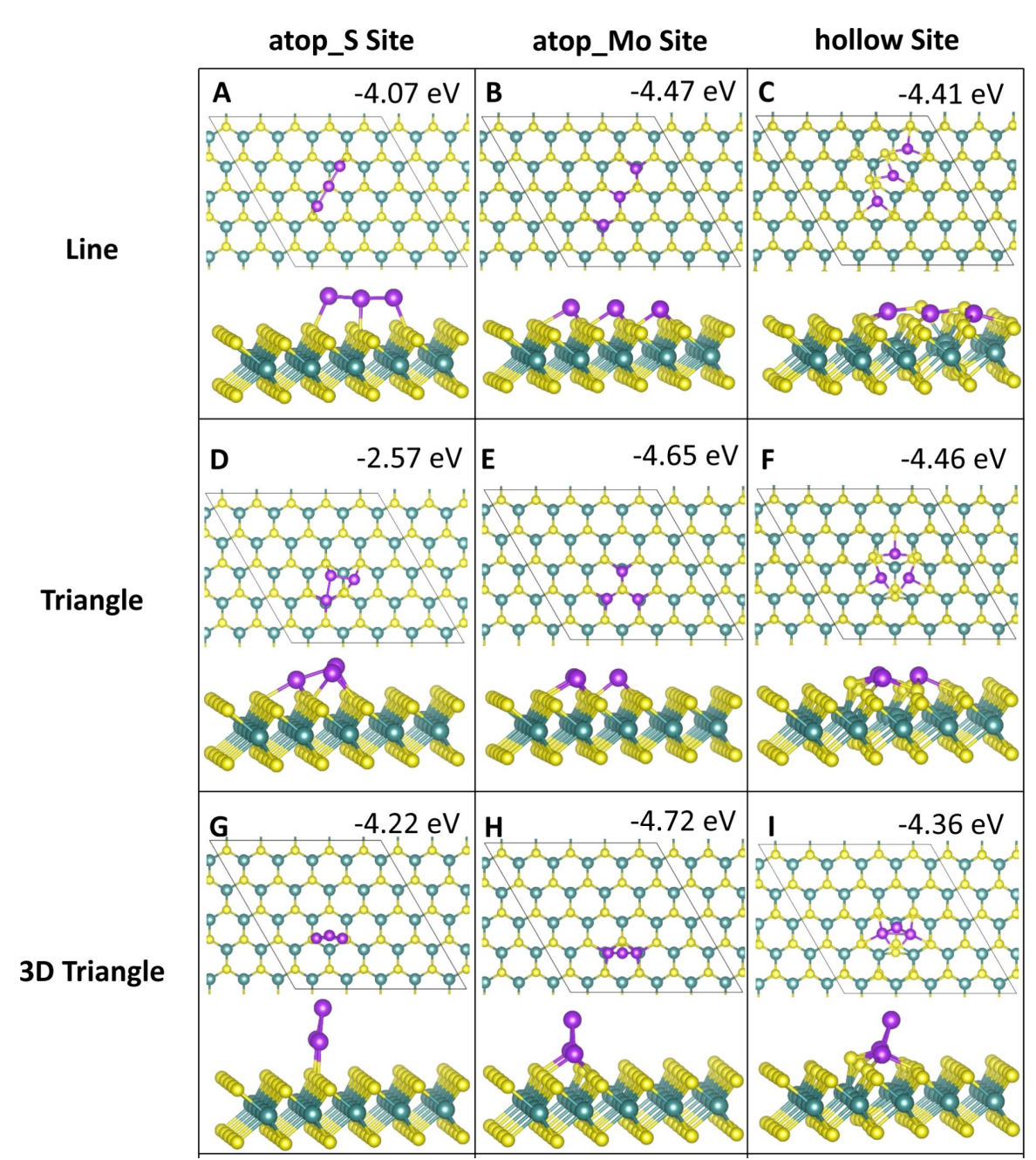

Figure 6: Atomic structure and energies of $\mathrm{Ru}_{3}$ adsorption modes on perfect $\mathrm{MoS}_{2}$.

the three adatoms are adsorbed in a line along neighbouring, equivalent sites. In the other two configurations the adatoms are arranged in a triangle, one $2 \mathrm{D}$ on the surface and the other in a $3 \mathrm{D}$ triangle; in the latter, the third adatom sits atop the two other adatoms.

The most favourable configuration for $\mathrm{Co}_{3}$ is the $2 \mathrm{D}$ triangle at site atop_Mo with a computed adsorption energy of $-6.13 \mathrm{eV} /$ atom (Figure 5E). The line and 3D triangle configurations are, however, competitive in energy. The line configuration is more favourable by between 0.01 and $0.32 \mathrm{eV}$ depending on the initial site at which the Co atoms bind. The $2 \mathrm{D}$ triangle with Co atoms at site atop_S has the least favourable adsorption energy of $-4.22 \mathrm{eV} /$ atom (Figure 5D). This configuration also has the weakest metal-metal interaction energy of $-0.98 \mathrm{eV}$, as the Co atoms move away from each other. The Co-Co distances are $2.08 \AA$ between the adjacent Co atoms and 4.89 to $4.98 \AA$ to the separated atom.
The largest metal-metal interaction energies, all of which are greater than $4 \mathrm{eV}$ in magnitude, can be observed for all 3D triangle configurations (Figure 5G-I) as well as for the line configuration at site atop_S (Figure 5A). This is due to the presence of $\mathrm{Co}-\mathrm{Co}$ bonds in all of these configurations, as there is no metal-metal bonding observed in the other structures. The Co-Co distances are between 2.20 and $2.25 \AA$, with some few longer bonds of approx. $2.6 \AA$ compared to $2.48 \AA$ in bulk $\mathrm{Co}[52]$.

For $\mathrm{Ru}_{3}$, a different trend is seen. Here, the most favourable adsorption occurs for the $3 \mathrm{D}$ triangle configuration with the $\mathrm{Ru}$ atoms at site atop_Mo (Figure $6 \mathrm{H}$ ). However, all $\mathrm{Ru}_{3}$ configurations, with the exception of the $2 \mathrm{D}$ triangle configuration at site atop_S, with an adsorption energy of $-2.57 \mathrm{eV} /$ atom (Figure 6D), differ by no more than $0.7 \mathrm{eV} /$ atom from this. This least favourable adsorption structure displays $\mathrm{Ru}$ atoms that migrate significantly from their original adsorption sites. While 
one atom remains at site atop_S, the second atom bridges between two S atoms between site atop_Mo and site hollow. The third atom has migrated to bind at site atop_Mo. Even though there are $\mathrm{Ru}-\mathrm{Ru}$ bonds, this structure is distorted, compared to the more stable structures, leading to a less favourable adsorption energy.

Compared to $\mathrm{Co}_{3}$, the metal-metal interactions are not as strong for $\mathrm{Ru}_{3}$. Similarly to $\mathrm{Co}_{3}$, the strongest metal-metal interactions are found for those structures where $\mathrm{Ru}-\mathrm{Ru}$ bonding is present. Similar to $\mathrm{Ru}_{2}$, adsorption in a linear configuration, at site hollow (Figure 6C), yields a positive interaction energy. The reason for this are the significant distortions to the ML, in which S atoms migrate out of the surface. Similar rearrangements can be observed for all $\mathrm{Ru}_{3}$ structures at site hollow (Figure 6C,F,I) as well as for the $2 \mathrm{D} \mathrm{Co}_{3}$ structures at site hollow (Figure 5C,F). Compared to copper, where we did not observe this distortion, the smaller size of Co and Ru compared to $\mathrm{Cu}$ may promote these distortions when the metal atoms are adsorbed above the hollow site hollow. In response the $\mathrm{S}$ atoms can migrate and rearrange to accommodate the additional metal atom.

\section{Four-atom adsorption}

For four-atom adsorption, four different cluster geometries were explored. There are two 2D clusters and two 3D clusters. The relaxed geometries are shown in Figure 7 and Figure 8. The 2D structures are four atoms adsorbed in a line along equivalent sites and in a rhombus shape with metal atoms binding to adjacent, equivalent sites. For the 3D structures, two atoms are adsorbed at equivalent sites, with two atoms atop these to create a $3 \mathrm{D}$ rectangle. Similarly, for the tetrahedral geometry, three atoms are adsorbed to the ML, with a fourth atom atop these to create a tetrahedron.

Adsorption of Co at site atop_Mo in the 3D tetrahedral configuration (Figure $7 \mathrm{G}, \mathrm{H}, \mathrm{K}$ ) is the most favourable geometry with a binding energy of $-6.33 \mathrm{eV} / \mathrm{atom}$, with a linear adsorption mode at site atop_Mo (Figure 7B) and a 3D tetrahedral configuration at site hollow (Figure 7L) showing binding energies of -6.10 and $-6.05 \mathrm{eV}$, respectively. Generally, the 3D rectangle structure is not as favourable, so that the 3D tetrahedral motifs predominate for four-atom clusters, with the $2 \mathrm{D}$ linear configurations also favourable.

Of the twelve initial binding configurations, upon relaxation five structures show atoms migrating from their original positions. Three of these are the 3D rectangle configuration, which shows that this geometry is not favourable for $\mathrm{Co}_{4}$. At sites atop_S and atop_Mo (Figure 7G,H), this configuration rearranges to the most favourable tetrahedral geometry with the base atoms adsorbed at site atop_Mo. At site hollow, one of the atop atoms moves to the surface to adsorb at a site atop_S, while the second atop atom migrates to rest between the two base atoms, which remain at site hollow, forming a 3D rhombus structure shown in Figure 7I. The remaining rearrangements are discussed in section S1.1.1 of Supporting Information File 1.

Rearrangements of the $\mathrm{MoS}_{2} \mathrm{ML}$ can also be observed. In the 2D configurations we can clearly see the Co atoms integrating into the S layer of the ML (Figure 7C,F,L). Co atoms from the line configuration at site hollow incorporate into the $\mathrm{S}$ layer with little movement away from the original lattice, which could be one of the origins for the relative stability of this structure.

For $\mathrm{Ru}_{4}$ adsorption, we find that similar to $\mathrm{Co}_{4}$, a 3D tetrahedral configuration at site atop_Mo is the most favourable geometry with an adsorption energy of $-5.14 \mathrm{eV}$ (Figure 8K). Similarly to $\mathrm{Co}_{4}$ and $\mathrm{Ru}_{3}$, five of the twelve $\mathrm{Ru}_{4}$ configurations exhibit atom migration to different sites or cause the ML to rearrange, or in some cases both.

However, in contrast to $\mathrm{Co}_{4}$, the tetrahedral configuration is not favoured in these rearrangements. Instead, we observe more structural distortions to the $\mathrm{Ru}_{4}$ geometries and the $\mathrm{MoS}_{2}$ lattice. In the case of the 3D rectangle at site atop_S these distortions cause a transition from a 3D structure to a 2D structure, as shown in Figure 8G. As for adsorptions at site hollow discussed previously, the most dramatic distortions to the $\mathrm{MoS}_{2}$ ML occur at this site. The line and tetrahedral configurations (Figure 8C,L), in particular, caused strong lattice distortions. While the $\mathrm{Ru}$ atoms have remained at their original adsorption sites, several S atoms migrated out of the surface to accommodate their incorporation into the $\mathrm{S}$ layer and have formed bonds with the $\mathrm{Ru}$ adatoms, creating a $\mathrm{Ru}_{4} \mathrm{~S}_{3}$ cluster. More details on the various geometries are given in section S1.1.2 of Supporting Information File 1.

\section{Discussion}

Comparing the contributions of metal-metal and metal-substrate interaction energies to the total binding energy is an important way of determining a preference towards $2 \mathrm{D}$ or $3 \mathrm{D}$ growth. With this particular system it is also important to consider that the metal-substrate interaction and the total binding energy reflect any energy changes associated with rearrangements in the $\mathrm{MoS}_{2} \mathrm{ML}$. With this in mind there are three different factors that can influence the metal-substrate interaction. These are (a) a lack of metal-metal bonds, (b) ML rearrangements including incorporation of metal atoms into the $S$ layer and (c) formation of metal-S clusters. Incorporation of 


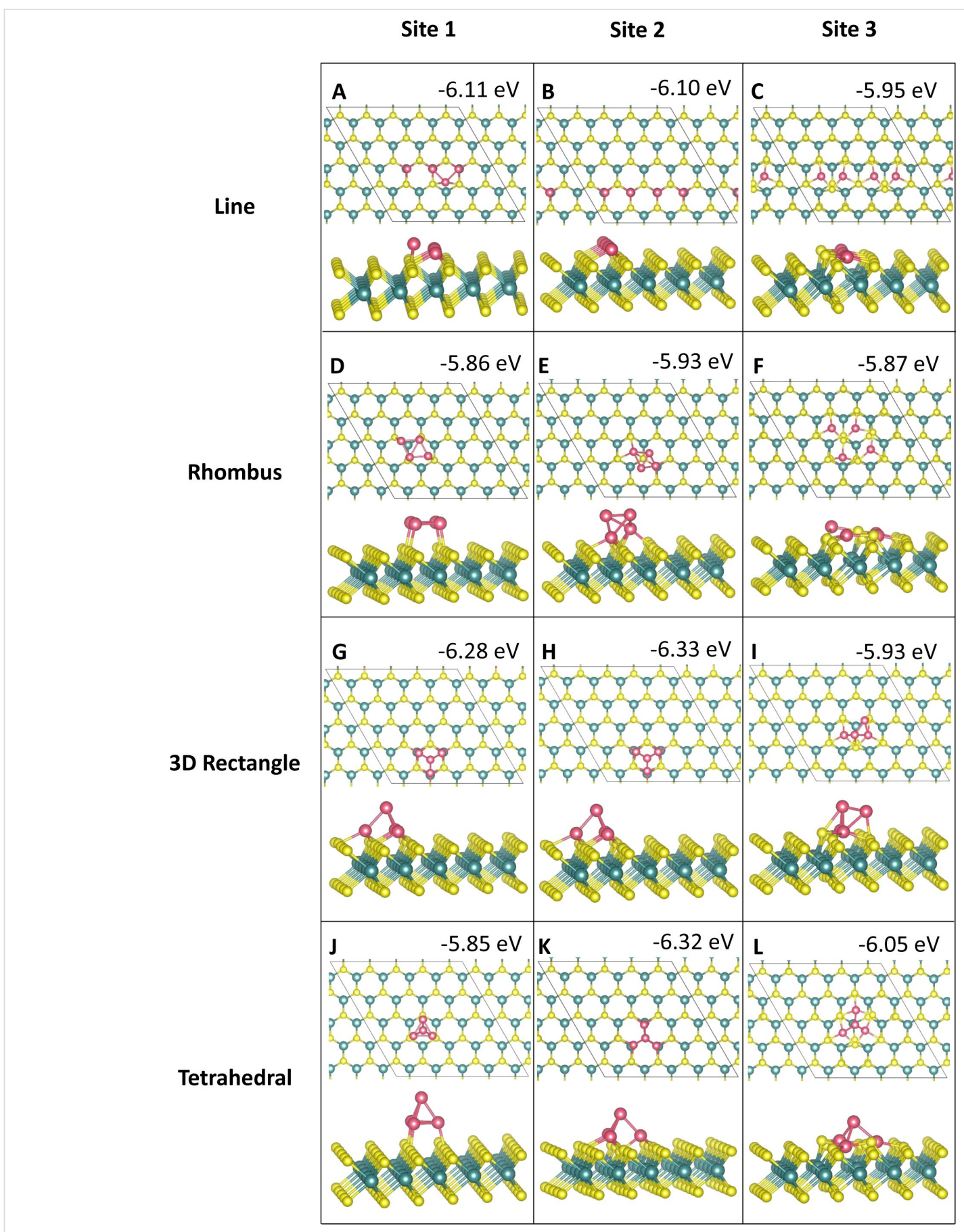

Figure 7: Atomic structure and energies of $\mathrm{Co}_{4}$ adsorption modes on perfect $\mathrm{MoS}_{2}$.

adatoms into the surface layer is known to increase the metal-substrate interaction, as is the lack of metal-metal bonds [48]. A gain in the metal-substrate interaction energy from formation of a metal-S cluster is due to Equation 2 not accounting for this, meaning that energy contributions from the formation of metal-S clusters are included in the metal-substrate interaction energy. Thus, it cannot be used in a straightforward manner as an indicator of 2D-vs-3D growth for these geometries.
We find that there are five $\mathrm{Co}_{n}$ geometries where the metal-substrate interaction is more favourable than the metal-metal interaction. These are the separated $\mathrm{Co}_{2}$ atoms at site atop_Mo along with all of the linear site hollow adsorptions for $\mathrm{Co}_{3}$ and $\mathrm{Co}_{4}$ clusters. For these structures the increase in the metal-substrate interaction is caused by the lack of metal-metal bonds. For the site hollow structures, the increase in the metal-substrate interaction is due to the incorporation of 


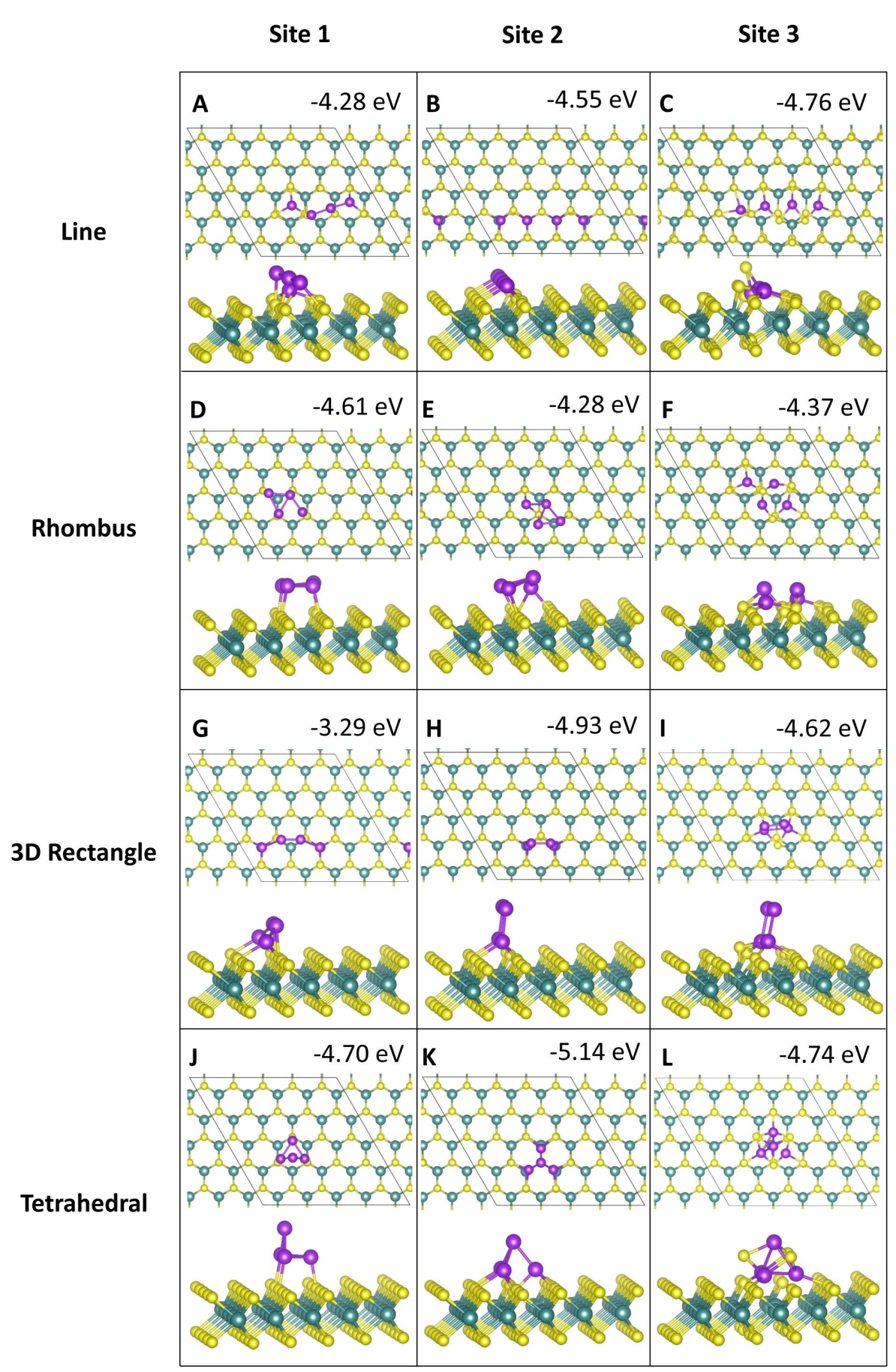

Figure 8: Atomic structure and energies of $\mathrm{Ru}_{4}$ adsorption modes on perfect $\mathrm{MoS}_{2}$.

Co into the $\mathrm{S}$ layer as well as the limited metal-metal interactions in a linear structure.

There are ten $\mathrm{Ru}_{n}$ structures where the metal-substrate interaction is more favourable than the metal-metal interaction. However, we observe significant ML distortions for $\mathrm{Ru}_{n}$ adsorption. Hence, this should not be used as the only indicator for poten- tial 2D-vs-3D growth. In these cases, both the metal-substrate and the metal-metal interactions were positive.

Similar to $\mathrm{Co}_{n}$, the majority of the structures that have a more favourable metal-substrate interaction are those that do not involve any metal-metal bonds or those where the Ru adatoms were incorporated into the $\mathrm{S}$ layer. Several of these structures 
involved the formation of metal-S structures, which can account in part for the larger number of structures with a more favourable metal-substrate interaction.

Bond length analysis shows that the length of Co-Co and $\mathrm{Ru}-\mathrm{Ru}$ bonds is directly influenced by the geometry of the adsorbed structure. This means that the majority of metal-metal bonds are shorter than the bulk metal-metal distances of $2.48 \AA$ [52] for Co and $2.67 \AA$ [55] for Ru, with some configurations showing longer distances depending on the arrangement of Co and $\mathrm{Ru}$ atoms. $\mathrm{Co}-\mathrm{S}$ and $\mathrm{Ru}-\mathrm{S}$ bonds change in length depending on the adsorption site, with the shortest bonds measured for site atop_S adsorptions. Bonds can also be longer due to structural distortions. Mo-S bonds are generally unaffected or slightly shorter than their equivalent on the bare ML. However, the local distortions described above can cause Mo-S distances to change by between -0.17 and $+0.28 \AA$ for $\mathrm{Co}$ adsorption and by between -0.26 and $+0.23 \AA$ for Ru adsorption. Section S1.5 of Supporting Information File 1 presents a more detailed analysis of the bonding.

Calculating addition energies allows us to study the energy gain as more atoms are added to a structure. Due to the many rearrangements of configurations observed, the energy gain computed from Equation 4 also contains the energy gained from the local atomic rearrangements previously described.

We observe particularly large addition energies for structures such as the $\mathrm{Co}_{4}$ line and $3 \mathrm{D}$ rectangle configurations, when rearrangements occur to realise a particularly favourable structure. Smaller addition energies are observed for rearrangements to a structure that is less favourable. Where no rearrangement occurs, the addition energies are of similar magnitude for different configurations, indicating that the variation arises only from the structure. Further details on addition energy are given in section S1.2 of Supporting Information File 1.

We now discuss the electronic properties of $\mathrm{Co}$ and $\mathrm{Ru}$ clusters adsorbed on $\mathrm{MoS}_{2}$. From the computed Bader charges, a metallic Co atom has 9.0 valence electrons, while a Co atom is considered oxidised when it has a Bader charge of less than 9.0 electrons. Similarly, metallic Ru has 8.0 valence electrons, and oxidised $\mathrm{Ru}$ will have a Bader charge of less than 8.0 electrons.

Analysis of the Bader charges for Co adsorption shows that, in general, atoms bound to the $\mathrm{MoS}_{2} \mathrm{ML}$ are oxidised. Adatoms that are only bound to other Co atoms remain metallic. Overall oxidation of Co atoms varies with atom coordination.

For Ru we find that atoms are partially oxidised when adsorbing to the $\mathrm{MoS}_{2} \mathrm{ML}$, with a computed Bader charge in the range of
7.6 to 7.8 electrons, compared to metallic Ru with 8.0 electrons. As the number of metal adatoms increases, atoms tend to be less oxidised and show more metallic character. Any Ru atom in a 3D configuration that is only adsorbed to other $\mathrm{Ru}$ atoms remains metallic with Bader charges of 7.9 to 8.0 electrons. The tetrahedral configuration at site hollow, which has formed a $\mathrm{Ru}_{4} \mathrm{~S}_{3}$ cluster has a Bader charge of 7.6 electrons for the atop $\mathrm{Ru}$ atom that forms new $\mathrm{Ru}-\mathrm{S}$ bonds. Further details of the Bader charge analysis are given in section S1.3 of Supporting Information File 1.

The changes in charge density are localised around the adatoms and the Mo and S atoms in the immediate neighbourhood of the adatoms. Atoms that were found to be near metallic during the Bader analysis are also found to have somewhat less charge density compared to atoms that were oxidised. There is no distinct difference in how $\mathrm{Co}$ and $\mathrm{Ru}$ affect the charge density with adsorption to $\mathrm{MoS}_{2}$. Examples of the charge density differences are shown in Figure S1 and Figure S2 of Supporting Information File 1, with some additional discussion in section S1.4.

Analysis of DOS plots shows that the Mo d-orbital or the S p-orbital contributions are largely unaffected by adatom adsorption. The metal d-orbital contribution increases for both Co and $\mathrm{Ru}$ as more adatoms are added, causing the total DOS to become increasingly more metallic compared to bare $\mathrm{MoS}_{2}$, which is a semiconductor. Metal d-orbital states appear in the bandgap for as little as a single adatom. These increase in magnitude as the overall metal contribution increases with added adatoms. Some mid-gap states for Mo d-orbitals and S p-orbitals also begin to appear as more adatoms are added, contributing to the increasingly metallic nature of the system. DOS plots for all configurations are shown in section S3 of Supporting Information File 1.

A brief analysis of the magnetism of Co structures is given in section S5 of Supporting Information File 1.

\section{Adsorption of $\mathrm{Co}$ and $\mathrm{Ru}$ clusters on defective $\mathrm{MoS}_{2}$}

It is well known from both theoretical and experimental studies, that the $\mathrm{MoS}_{2} \mathrm{ML}$ easily forms $\mathrm{S}$ vacancies. To get a first insight into how the presence of such a vacancy might change the interaction between the metal and the ML, we repeat the simulations of the adsorption of single metal atoms and $\mathrm{M}_{4}$ structures on a defective $\mathrm{MoS}_{2} \mathrm{ML}$ with a single $\mathrm{S}$ vacancy. This was carried out before in our previous work with $\mathrm{Cu}$ adsorption. Using the formation of $\mathrm{H}_{2} \mathrm{~S}$ from $\mathrm{H}_{2}$, we computed a vacancy formation energy of $-6.16 \mathrm{eV}$, which confirms that defects are easily formed. Furthermore, our results showed that 
the presence of an $\mathrm{S}$ vacancy (giving a concentration of $2 \%$ vacancies per supercell) improved $\mathrm{Cu}$ adhesion and promoted the formation of 3D clusters [28].

The binding energies, the metal-substrate interaction energies, and the metal-metal interaction energies for all structures on defective $\mathrm{MoS}_{2}$ are shown in Table 4, Table 5, and Table 6, respectively. Discussion on bond lengths can be found in section S2.1 of Supporting Information File 1.

For single-atom adsorption, we find that the metal-ML interaction is stronger at site atop_S, where, similarly to $\mathrm{Cu}$ [28], Co and $\mathrm{Ru}$ fill the vacancy site. This leads to a stronger binding energy by approx. $2 \mathrm{eV}$ for Co and by approx. $3 \mathrm{eV}$ for $\mathrm{Ru}$, compared to the same initial adsorption site on the pristine ML. While, for $\mathrm{Cu}$ we found that the metal-ML interaction was en- hanced at all sites, for $\mathrm{Co}$ and $\mathrm{Ru}$ we find that the initial adsorption at sites atop_Mo and hollow is not stable. For Co, a single atom at either site atop_Mo or hollow migrates away from its original adsorption site and the vacancy to bridge between two S atoms between site atop_Mo and hollow. The same occurs for a Ru atom at site hollow, while adsorption at site atop_Mo leads to the atom migrating to the nearest site hollow. These adsorption configurations are less favourable by approximately $0.5 \mathrm{eV}$, than adsorption at the corresponding sites atop_Mo and hollow on the pristine ML, and more favourable than adsorptions at site atop_S on the pristine ML. The corresponding geometries are shown in Figure 9.

For $\mathrm{Co}_{4}$, a decrease in the interaction strength is observed for the line configuration at site atop_S, and the 3D rectangle at site atop_Mo. For the line configuration at site atop_S, this is

Table 4: Computed binding energies for $\mathrm{Co}_{1}, \mathrm{Co}_{4}, \mathrm{Ru}_{1}$ and $\mathrm{Ru}_{4}$ on defective $\mathrm{MoS}_{2} \mathrm{ML}$ for various atom configurations using Equation 1.

\begin{tabular}{|c|c|c|c|c|c|c|c|}
\hline \multirow{2}{*}{$\begin{array}{l}\text { No. of metal } \\
\text { atoms }\end{array}$} & \multirow[t]{2}{*}{ Configuration } & \multicolumn{3}{|c|}{$E_{\text {bind }} /$ Co-atom [eV] } & \multicolumn{3}{|c|}{$E_{\text {bind }} / \mathrm{Ru}$-atom [eV] } \\
\hline & & S_atop & Mo_atop & hollow & S_atop & Mo_atop & hollow \\
\hline 1 & - & -6.83 & -5.22 & -5.12 & -5.59 & -3.32 & -2.91 \\
\hline \multirow[t]{4}{*}{4} & line & -5.43 & -6.22 & - & -4.48 & -4.91 & -5.16 \\
\hline & rhombus & - & -6.25 & -6.64 & - & -4.83 & -4.33 \\
\hline & $3 \mathrm{D}$ rectangle & - & -5.78 & -6.29 & - & -4.89 & -4.71 \\
\hline & tetrahedral & - & -6.22 & -6.00 & - & -4.95 & -4.72 \\
\hline
\end{tabular}

Table 5: Computed metal-substrate interaction energies $\mathrm{Co}_{4}$ and $\mathrm{Ru}_{4}$ on defective $\mathrm{MoS}_{2} \mathrm{ML}$ for various atom configurations using Equation 2.

\begin{tabular}{|c|c|c|c|c|c|c|c|}
\hline \multirow{2}{*}{$\begin{array}{l}\text { No. of metal } \\
\text { atoms }\end{array}$} & \multirow[t]{2}{*}{ Configuration } & \multicolumn{3}{|c|}{$E_{\text {metal-substrate }} /$ Co-atom [eV] } & \multicolumn{3}{|c|}{$E_{\text {metal-substrate }} /$ Ru-atom [eV] } \\
\hline & & S_atop & Mo_atop & hollow & S_atop & Mo_atop & hollow \\
\hline \multirow[t]{4}{*}{4} & line & -1.79 & -2.30 & - & -0.16 & -2.68 & -4.52 \\
\hline & rhombus & - & -1.96 & -4.15 & - & -2.76 & -2.88 \\
\hline & $3 \mathrm{D}$ rectangle & - & -0.73 & -1.58 & - & -1.01 & -0.70 \\
\hline & tetrahedral & - & -1.37 & -1.17 & - & -1.60 & -1.06 \\
\hline
\end{tabular}

Table 6: Computed metal-metal interaction energies for $\mathrm{Co}_{4}$ and $\mathrm{Ru}_{4}$ on defective $\mathrm{MoS}_{2}$ using Equation 3.

\begin{tabular}{|c|c|c|c|c|c|c|c|}
\hline \multirow{2}{*}{$\begin{array}{l}\text { No. of metal } \\
\text { atoms }\end{array}$} & \multirow[t]{2}{*}{ Configuration } & \multicolumn{3}{|c|}{$E_{\text {interact }} /$ Co-atom [eV] } & \multicolumn{3}{|c|}{$E_{\text {interact }} / \mathrm{Ru}$-atom [eV] } \\
\hline & & S_atop & Mo_atop & hollow & S_atop & Mo_atop & hollow \\
\hline \multirow[t]{4}{*}{4} & line & -4.05 & -3.92 & - & -2.35 & -2.22 & -0.64 \\
\hline & rhombus & - & -4.29 & -2.49 & - & -2.07 & -1.45 \\
\hline & $3 \mathrm{D}$ rectangle & - & -5.05 & -4.71 & - & -3.88 & -4.00 \\
\hline & tetrahedral & - & -4.86 & -4.83 & - & -3.35 & -3.67 \\
\hline
\end{tabular}



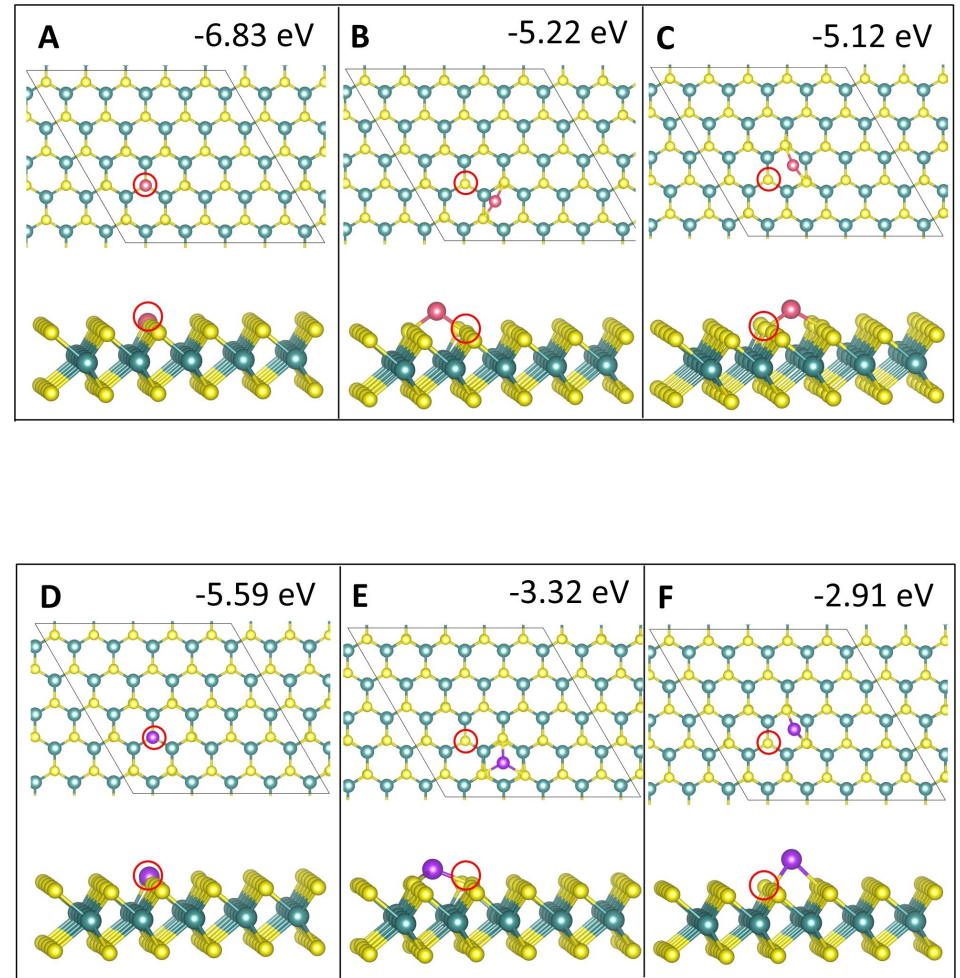

Figure 9: Adsorption modes of single $\mathrm{Co}$ (pink) and Ru (purple) atoms on defective $\mathrm{MoS}_{2}$. The red circle indicates the location of the $\mathrm{S}$ vacancy.

due to the atoms remaining in a line, compared to the migration to a different site that was observed on the pristine surface. Despite the formation of a bond between two of the Co atoms and one of the Co atoms migrating into the vacancy, the binding energy of $-5.43 \mathrm{eV}$ is less favourable compared to $-6.11 \mathrm{eV}$ on the pristine surface. All geometries for $\mathrm{Co}_{4}$ are shown in Figure 10.

An increase in the interaction strength is observed for the line and rhombus configurations at site atop_Mo and the rhombus and $3 \mathrm{D}$ rectangle configurations at site hollow. Depending on the configuration this change can be attributed to bond formation, structural rearrangement compared to the same adsorption configuration on the pristine ML, or in the case of the rhombus at site hollow, surface distortion and $\mathrm{C}_{x} \mathrm{~S}_{y}$ formation, which also creates an additional $\mathrm{S}$ vacancy. The latter is facilitated by the presence of the vacancy and does not occur on the pristine ML.

The presence of the vacancy changes the charge distribution compared to the pristine surface. Atoms near the vacancy are less oxidised (with computed Bader charges of 8.7 to 8.8 electrons) and atoms further away from the vacancy site are more oxidised (Bader charges of 8.5 electrons). The apex atoms in 3D structures are metallic, even for structures where these were slightly oxidised on the pristine surface.

Even though the effects of the vacancy and the adsorption of Co remain localised, the charge density difference shows that the area over which significant changes in charge density take place is larger than on the pristine surface. Visualisations of the charge density differences on the defective ML are included in Figure S1 and Figure S2 of Supporting Information File 1. Metal-metal interaction energies for $\mathrm{Co}$ are found to be generally of the same magnitude as on the pristine surface. There is no clear correlation between the binding energy and the metal-metal interaction energy. There are no Co geometries on the defective surface where the metal-substrate interaction is more favourable than the metal-metal interaction. The interaction energy is also not affected by the incorporation of S into the Co cluster as is observed for the rhombus configuration at site hollow. This structure has very favourable adhesion to the ML, but the metal-metal interaction energy is similar to that on the pristine surface.

$\mathrm{Ru}_{4}$ structures remained largely unchanged compared to the pristine ML. All $\mathrm{Ru}_{4}$ geometries are shown in Figure 11. The 


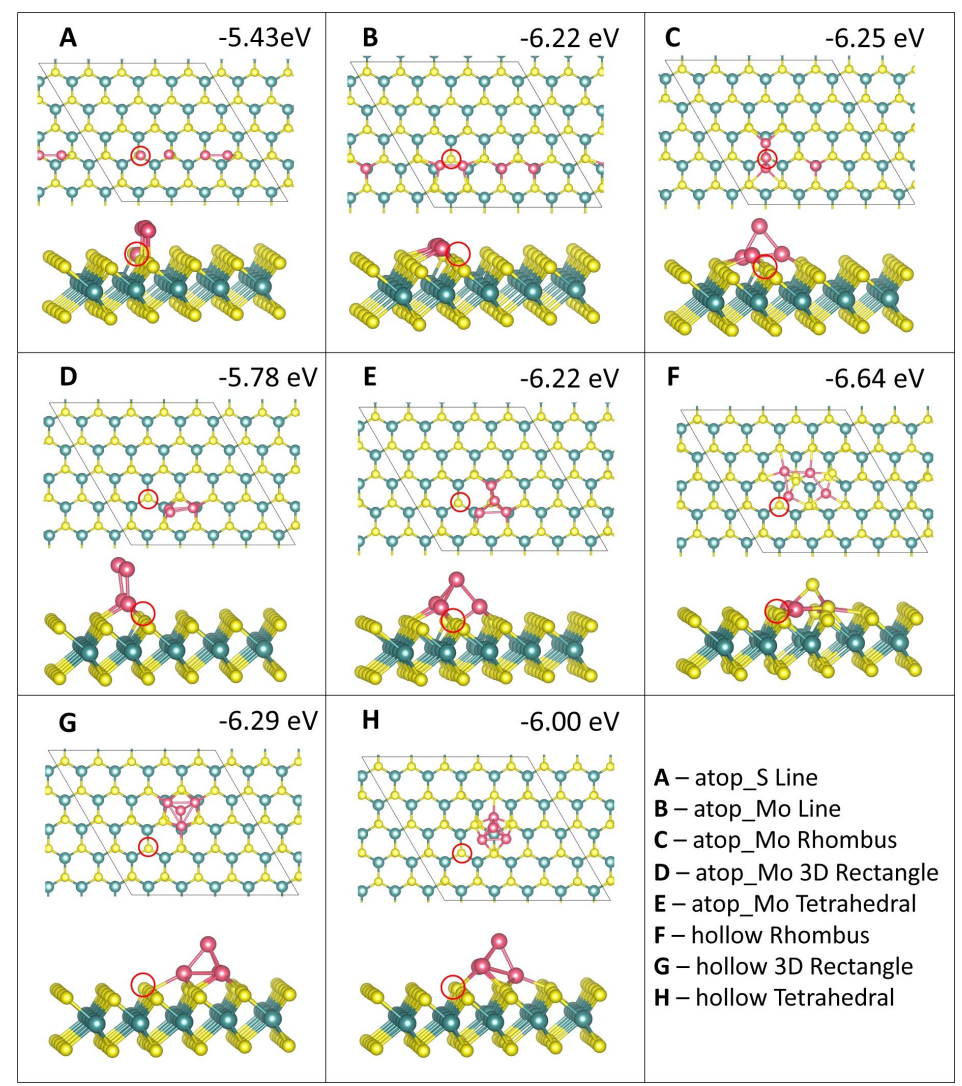

Figure 10: Atomic structure and energies of $\mathrm{Co}_{4}$ adsorption modes on defective $\mathrm{MoS}_{2}$. The red circle indicates the location of the $\mathrm{S}$ vacancy.

only decrease in stability is observed for the tetrahedron at site atop_Mo, which distorts away from the vacancy. An increase in stability of between 0.1 and $0.4 \mathrm{eV}$ is observed for the line configurations at sites atop_S and atop_Mo, the rhombus configuration at site atop_Mo and the 3D rectangle configuration at site hollow.

The line configuration initially at site atop_S relaxes to produce two separated $\mathrm{Co}_{2}$ clusters. Of the two atoms near the vacancy, one has moved into the vacancy to replace $S$, while the other is adsorbed at a site hollow. The energy gain compared to the pristine surface can be attributed to the additional $\mathrm{Ru}-\mathrm{Ru}$ bonds as well as the incorporation of Ru into the vacancy, which is always favourable. The line configuration at site atop_Mo has the same geometry for $\mathrm{Ru}_{4}$ as $\mathrm{Co}_{4}$ and $\mathrm{Cu}_{4}$ [28], with two $\mathrm{Ru}$ atoms bridging over the vacancy, as shown in Figure 11B. Similarly, the rhombus configuration at site atop_Mo has rearranged to the same 3D triangle over the vacancy with the fourth atom at the nearest site atop_Mo that was observed for $\mathrm{Co}_{4}$ and $\mathrm{Cu}_{4}$ on the defective ML.

Even though the binding energy for the tetrahedron at site hollow is similar on the defective ML and the pristine ML, the presence of the vacancy prevents the incorporation of the basal $\mathrm{Ru}$ atoms into the $\mathrm{S}$ layer. This is the case for all the structures at site hollow, which cause less distortion on the defective ML compared to the pristine ML. The exception to this is the line configuration at the hollow site (Figure $11 \mathrm{~F}$ ). Here, two $\mathrm{Ru}_{2}$ dimers are formed, with a $\mathrm{S}$ atom bridging between them, creating a $\mathrm{Ru}_{4} \mathrm{~S}$ structure and an additional vacancy in the ML. This is also the most favourable $\mathrm{Ru}_{4}$ structure on the defective ML.

$\mathrm{Ru}$ atoms are similarly oxidised on both the pristine and the defective ML, although as for Co, atoms near the vacancy tend to be less oxidised with approx. 7.8 electrons. Once again, atop $\mathrm{Ru}$ atoms in $3 \mathrm{D}$ structures remain metallic, with computed Bader charges of 7.9 to 8.0 electrons. Interestingly, the atop atom in the rearranged rhombus structure at site atop_Mo is reduced, with a computed Bader charge of 8.1 electrons, making it the only metal atom in any of the configurations to become reduced. Additional Bader analysis is detailed in section S2.2 of Supporting Information File 1. The charge density difference for $\mathrm{Ru}$ on the defective $\mathrm{ML}$ is similar to that on the pristine ML (Figure S2G,H of Supporting Information File 1). 


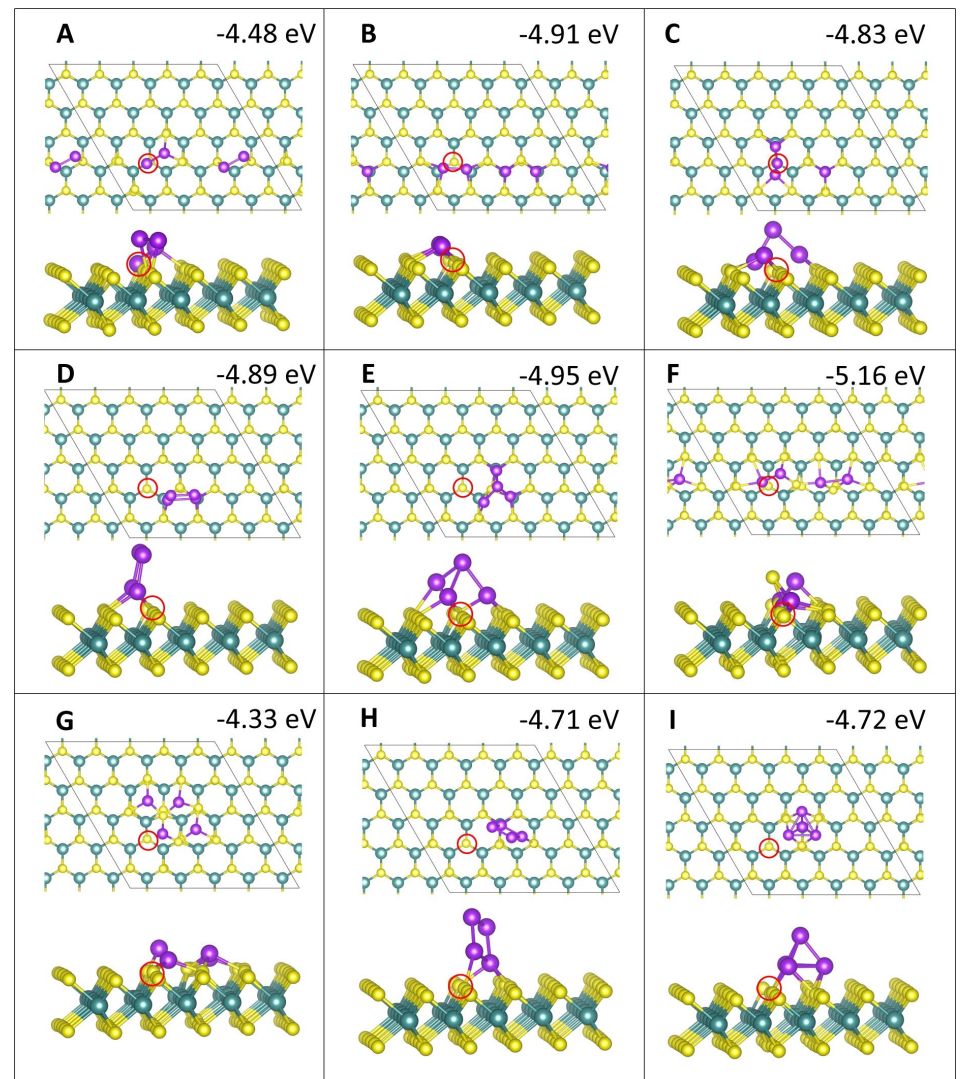
A - atop_S Line
B - atop_Mo Line
F - hollow Line
C - atop_Mo Rhombus
G - hollow Rhombus
H-hollow 3D Rectangle
E - atop_Mo Tetrahedral

Figure 11: Atomic structure and energies of $\mathrm{Ru}_{4}$ adsorption modes on defective $\mathrm{MoS}_{2}$. The red circle indicates the location of the $S$ vacancy.

The effect of a vacancy on the metal-metal interaction energy of adsorbed Ru structures is significant. For most structures, the magnitude of the metal-metal interaction energies is approximately half that of the pristine ML. This is best illustrated using the example of the rearranged rhombus configuration at site atop_Mo as this same rearrangement also occurs for Co. While for Co the interaction energy weakens by approximately $0.7 \mathrm{eV}$ due to the separated fourth atom, for Ru the interaction is weakened by more than $3 \mathrm{eV}$ from $-5.29 \mathrm{eV}$ on the pristine surface to $-2.07 \mathrm{eV}$ on the defective surface. This change can be attributed to the effect of the vacancy on adsorbed Ru structures. There are some exceptions, where the metal-metal interaction is strengthened compared to the pristine ML. However, this is due to a difference in geometry compared to the pristine surface.

There are three geometries adsorbed on the defective ML that have a metal-substrate interaction more favourable than the metal-metal interaction. These are the linear configurations at site atop_Mo and hollow and the rhombus at site hollow. All three of these structures have none or few metal-metal bonds and both configurations at site hollow involve incorporation into the S layer, which increases the strength of the metal-substrate interaction.

\section{Comparison of interactions of $\mathrm{Cu}, \mathrm{Co}$, and $\mathrm{Ru}$ with pristine and defective $\mathrm{MoS}_{2}$}

The interaction of the metals $\mathrm{Cu}, \mathrm{Co}$, and $\mathrm{Ru}$ with the pristine and defective $\mathrm{MoS}_{2}$ ML clearly depends on the nature of the metal. This is most obvious from the magnitude of the binding energies of a single atom. A single $\mathrm{Cu}$ atom binds to pristine $\mathrm{MoS}_{2}$ with energies ranging from -0.8 to $-1.3 \mathrm{eV}$. The adsorption energy of a Co atom ranges between -4.2 and $-5.8 \mathrm{eV}$, while the adsorption energy of a $\mathrm{Ru}$ atom ranges between -2.5 and $-3.9 \mathrm{eV}$.

For metal atoms and dimers, the stability of $\mathrm{Cu}$ and $\mathrm{Ru}$ adsorption is governed by the adsorption site, with site atop_Mo the 
most favourable site for both. Ru atoms at site hollow cause distortions in the ML. For Co, the adsorption site can also influence the stability, with site atop_Mo the most favourable overall. Site atop_Mo is favoured, as it follows the $\mathrm{MoS}_{2}$ lattice structure. We find that this, as well as the symmetrical addition of atoms at the same site instead of non-equivalent sites, is correlated with the strength of adhesion.

As $n$ increases, $\mathrm{Cu}$ adsorption no longer depends on the adsorption site. Instead, the stability of structures is governed by the number of $\mathrm{Cu}-\mathrm{Cu}$ bonds. This means that for $n=3-4$, all geometries have very similar binding energies. Further, for $n=4$, site atop_S is the most favourable. The distance between neighbouring sites atop_S is shorter compared to sites atop_Mo and hollow and the proximity of atoms adsorbed at site atop_S facilitates $\mathrm{Cu}-\mathrm{Cu}$ bond formation [28].

Co adsorption continues to be controlled by the adsorption site for $n=3$. Different geometries have very similar energies at the same site, with the most favourable adsorption configurations at site atop_Mo. $\mathrm{Co}_{4}$ prefers tetrahedral geometries on pristine $\mathrm{MoS}_{2}$, with the majority of adsorption geometries rearranging to this type of structure. Some of the $2 \mathrm{D}$ structures at site hollow result in strong distortions, involving metal migration into the $\mathrm{S}$ layer, which increases the strength of their interaction with the ML and makes these structures competitive in energy with the tetrahedral geometries.

Similarly to $\mathrm{Co}, \mathrm{Ru}_{3}$ adsorption is mainly influenced by the adsorption site, with some influences from the overall geometry. This is particularly noticeable at site atop_S, where the triangle configuration has a binding energy that is $1.5 \mathrm{eV}$ weaker compared to the other two geometries. This is also clear evidence that $\mathrm{Ru}$ atoms prefer to be associated, as the lower binding energy for the triangle at site atop_S arises from the separation of the atoms. In contrast, when $n=4$, Ru structures are more stable when there are minimal distortions to the geometry or the ML, or when Ru atoms incorporate into the $\mathrm{S}$ layer and $\mathrm{Ru}_{x} \mathrm{~S}_{y}$ structures are formed.

For $\mathrm{Cu}, \mathrm{Co}$, and $\mathrm{Ru}$, adsorption of a single atom on the defective ML is most favourable when the metal atom fills the vacancy. $\mathrm{Cu}$ adsorption at sites atop_Mo and hollow is also enhanced, while these adsorption sites become unfavourable for $\mathrm{Co}$ and $\mathrm{Ru}$ as the adatoms migrate away from the vacancy during the relaxation. Adsorption at the bridge site for Co and at the bridge site and site hollow for $\mathrm{Ru}$ is less favourable than adsorption on the pristine ML.

The presence of the S vacancy also enhances the binding of $\mathrm{Cu}_{4}$, although only a limited number of geometries were stable, while others were repelled from the ML. The stable configurations showed a preference to be $3 \mathrm{D} .2 \mathrm{D}$ and $3 \mathrm{D} \mathrm{Co}_{4}$ structures were competitive in energy, with a preference towards structures that incorporated into the $\mathrm{S}$ layer. Further, the vacancy facilitated the transfer of an $\mathrm{S}$ atom from the ML onto the Co structure to create a $\mathrm{Co}_{4} \mathrm{~S}$ cluster, which was also the most favourable $\mathrm{Co}_{4}$ structure on the defective ML. This shows that, in the presence of $\mathrm{Co}$, further vacancies can be formed through the transfer of $S$ atoms onto the metal cluster. In contrast, the presence of the vacancy prevented the incorporation of $\mathrm{Ru}$ atoms into the $\mathrm{S}$ layer to some extent and was also found to weaken metal-metal interactions. However, $\mathrm{a} \mathrm{Ru}_{2} \mathrm{~S}$ cluster with two adjacent $\mathrm{Ru}$ atoms was formed and is the most favourable structure, similar to $\mathrm{Co}_{4}$ adsorption.

It is difficult to predict whether a thin film will grow in a $2 \mathrm{D}$ or 3D structure, but a useful descriptor is how the metal-substrate interaction compares to the metal-metal interaction and how the total binding energy compares to the bulk metal cohesive energy. While the metal-substrate interaction exceeds the metal-metal interaction energy for several $\mathrm{Co}_{n}$ and $\mathrm{Ru}_{n}$ structures, only Co has a total binding energy that is more favourable than the cohesive energy. The most favourable adsorptions for both $\mathrm{Ru}$ and $\mathrm{Cu}$ are $1 \mathrm{eV}$ less favourable than their respective cohesive energies [58]. Despite this, we have shown that $\mathrm{Ru}$ incorporation into the $\mathrm{S}$ layer on the pristine surface and the presence of a vacancy both enhance the $\mathrm{Ru}$-substrate interaction and weaken the metal-metal interaction. It is therefore possible that the presence of more vacancies in the $\mathrm{MoS}_{2} \mathrm{ML}$ could promote a 2D growth of Ru. Further work including the calculation of the activation energies for $2 \mathrm{D}$ or $3 \mathrm{D}$ clusters will give a more detailed insight into the processes that control aggregation on the surface. However, this is out of the scope of the current study.

Based on our findings $\mathrm{MoS}_{2}$ would be most suitable as a barrier+liner for a Co interconnect, although based on our results, there are some concerns how the transfer of $\mathrm{S}$ atoms from the ML to $\mathrm{Co}_{n}$ would affect the purity of the interconnect. $\mathrm{Ru}$ on $\mathrm{MoS}_{2}$ might be better suited as a catalyst. However further studies involving larger $\mathrm{Ru}_{n}$ structures are needed to determine if the overall strength of the interaction between $\mathrm{Ru}$ and $\mathrm{MoS}_{2}$ could be enough to prevent agglomeration. As Ru was less likely to incorporate into the ML on a defective ML, growth of Ru on a defective $\mathrm{MoS}_{2} \mathrm{ML}$ could be suitable for $\mathrm{Ru}$ interconnect systems.

\section{Conclusion}

We have presented an extensive study of the interaction of $\mathrm{Co}_{n}$ and $\mathrm{Ru}_{n}$ species, with $n=1-4$, at a perfect and a defective $\mathrm{MoS}_{2}$ monolayer. We have also compared these metals to $\mathrm{Cu}_{n}$ 
from our earlier work. $\mathrm{MoS}_{2}$ is of great interest for the barrier layer in semiconductor devices and as a support in catalysis, while $\mathrm{Ru}$ and $\mathrm{Co}$ are potential replacements for $\mathrm{Cu}$ as the interconnect metal and are used in metal catalysis. Thus, understanding how the metals interact with $\mathrm{MoS}_{2}$ is important. We find that the stability of single-atom adsorption follows the trend $\mathrm{Co}>\mathrm{Ru}>\mathrm{Cu}$. Furthermore, this trend holds for the adsorption of all metal species, regardless of the number of adatoms. Co typically adsorbs more strongly than $\mathrm{Ru}$ to the $\mathrm{MoS}_{2} \mathrm{ML}$ by up to $2.0 \mathrm{eV}$, while adsorption is stronger by up to $5.0 \mathrm{eV}$ compared to $\mathrm{Cu}$.

For two- and three-atom nanoclusters on perfect $\mathrm{MoS}_{2}$, we find that the preferred adsorption configuration is determined by the adsorption site at the monolayer. $\mathrm{Ru}_{n}$ adsorption is accompanied by notable surface distortions in the monolayer, in particular the migration of sulfur atoms off their original sites as $\mathrm{Ru}$ atoms incorporate into the $\mathrm{S}$ layer of $\mathrm{MoS}_{2}$. Such rearrangements are not seen for $\mathrm{Cu}$ or $\mathrm{Co}$. The binding of $\mathrm{Cu}$ and $\mathrm{Co}$ nanoclusters appears to be driven by the formation of metal-metal and metal-surface bonds, whereas for $\mathrm{Ru}$, the adsorption configuration is the dominant factor.

The four-atom nanoclusters are the first clusters where 2D and $3 \mathrm{D}$ configurations can be compared. $\mathrm{Co}_{4}$ structures prefer to adsorb in tetrahedral 3D geometries, as is evident from the rearrangement of the atomic structure when Co atoms are close enough that $\mathrm{Co}-\mathrm{Co}$ bonds can form. However, linear configurations without $\mathrm{Co}-\mathrm{Co}$ bonds are competitive in energy with these tetrahedral geometries. This likely originates from the incorporation of Co atoms into the $\mathrm{S}$ layer in such structures. In turn, this indicates a strong interaction with the $\mathrm{MoS}_{2} \mathrm{ML}$, while tetrahedral geometries have an energy gain originating from $\mathrm{Co}-\mathrm{Co}$ interactions instead. If we compare with $\mathrm{Cu}$, the $\mathrm{Co}-\mathrm{S}$ bond enthalpy is much higher than the $\mathrm{Cu}-\mathrm{S}$ bond enthalpy, at $331 \mathrm{~kJ} / \mathrm{mol}$ and $274.5 \mathrm{~kJ} / \mathrm{mol}$ respectively (we did not find data for $\mathrm{Ru}-\mathrm{S}$ bonds), and the Co cohesive energy is larger at $4.39 \mathrm{eV}$, compared to $3.49 \mathrm{eV}$ for $\mathrm{Cu}$ [58].

$\mathrm{Ru}_{4}$ does not have the same preference for tetrahedral motifs as $\mathrm{Co}_{4}$, despite the tetrahedral configuration at site atop_Mo being the most favourable adsorption mode. Nevertheless, clustered geometries are preferred compared to linear adsorption, probably as a result of the larger Ru cohesive energy of $6.74 \mathrm{eV}$. Structures with minimal distortion are particularly favoured, as well as those where $\mathrm{Ru}$ incorporates into the $\mathrm{S}$ layer of $\mathrm{MoS}_{2}$ and $\mathrm{Ru}_{x} \mathrm{~S}_{y}$ clusters are formed.

On the defective ML, adsorption is most favourable at the vacancy site, where single metal atoms fill the missing $\mathrm{S}$ site, causing a significant increase in the interaction energy com- pared to the pristine ML. Adsorption at other sites is less favourable or unstable compared to the pristine ML. 2D and 3D $\mathrm{Co}_{4}$ structures are competitive in energy in the presence of a vacancy, with a preference towards those structures that have metal incorporation into the $\mathrm{S}$ layer. The vacancy also facilitates the transfer of $S$ atoms onto the Co cluster and thus the formation of additional vacancies.

The most favourable $\mathrm{Ru}_{4}$ structure on the defective $\mathrm{ML}$ is the line configuration at site hollow where transfer of an $\mathrm{S}$ atom onto the Ru structure has occurred. Despite this, we find that $\mathrm{Ru}$ atoms are less likely to incorporate into the $\mathrm{S}$ layer on a defective surface and that the metal-metal interaction energy is weakened, indicating that in the presence of more vacancies $2 \mathrm{D}$ growth of Ru should be promoted.

Our overall findings indicate that for Co 2D and 3D cluster adsorption structures are competitive. However, the binding energy of Co on $\mathrm{MoS}_{2}$ is significantly more favourable than the cohesive energy of Co. This strong binding energy as well as the favourable metal-substrate interaction should inhibit migration of atoms to form 3D structures during thin film deposition, resulting in a 2D film suitable for interconnect applications, without the need of an additional liner material to promote wetting. This is the subject of further work and will include study of the activation energies for $2 \mathrm{D}$ and $3 \mathrm{D}$ structures.

In contrast, $\mathrm{Ru}$ has a binding energy that is less favourable than its cohesive energy. This suggests that $3 \mathrm{D}$ growth $\mathrm{Ru}$ on $\mathrm{MoS}_{2}$ will be promoted, making this system more suitable for catalysis applications where 3D structures with large surface-tovolume ratios are desired. However, given the several structures for which the metal-substrate interaction is more favourable than the metal-metal interaction and the overall weakening of the metal-metal interactions caused by an $\mathrm{S}$ vacancy, a $2 \mathrm{D} \mathrm{Ru}$ thin film could be formed in the presence of $\mathrm{S}$ vacancies.

\section{Supporting Information}

Supporting Information features additional data on geometries, addition energies, Bader analysis, charge density difference, bondlengths, DOS, van der Waals interactions, and Co magnetism.

\section{Supporting Information File 1}

Additional experimental data.

[https://www.beilstein-journals.org/bjnano/content/ supplementary/2190-4286-12-56-S1.pdf] 


\section{Acknowledgements}

Access to SFI funded computing resources at Tyndall Institute and through the Irish Centre for High End Computing, ICHEC, is gratefully acknowledged.

\section{Funding}

We acknowledge funding by Science Foundation Ireland (SFI) through the SFI-NSF China Partnership Program, Grant No. 17/ NSFC/5279.

\section{ORCID ${ }^{\circledR}$ iDs}

Cara-Lena Nies - https://orcid.org/0000-0003-0959-2615 Michael Nolan - https://orcid.org/0000-0002-5224-8580

\section{Preprint}

A non-peer-reviewed version of this article has been previously published as a preprint: https://doi.org/10.26434/chemrxiv.14237336.v1

\section{References}

1. Samad, A.; Shafique, A.; Shin, Y.-H. Nanotechnology 2017, 28 , 175401. doi:10.1088/1361-6528/aa6536

2. Ye, C.; Chao, D.; Shan, J.; Li, H.; Davey, K.; Qiao, S.-Z. Matter 2020, 2, 323-344. doi:10.1016/j.matt.2019.12.020

3. Le, D.; Rawal, T. B.; Rahman, T. S. J. Phys. Chem. C 2014, 118, 5346-5351. doi:10.1021/jp411256g

4. Sharma, A.; Srivastava, A.; Husain, M.; Khan, M. S. J. Mater. Sci. 2018, 53, 9578-9588. doi:10.1007/s10853-018-2269-5

5. Ding, Y.; Wang, Y.; Ni, J.; Shi, L.; Shi, S.; Tang, W. Phys. B (Amsterdam, Neth.) 2011, 406, 2254-2260. doi:10.1016/j.physb.2011.03.044

6. Zahid, F.; Liu, L.; Zhu, Y.; Wang, J.; Guo, H. AIP Adv. 2013, 3, 052111. doi:10.1063/1.4804936

7. Lo, C.-L.; Smithe, K. K. H.; Mehta, R.; Chugh, S.; Pop, E.; Chen, Z. Atomically thin diffusion barriers for ultra-scaled $\mathrm{Cu}$ interconnects implemented by 2D materials. In 2017 IEEE International Reliability Physics Symposium (IRPS), 2017; MR-4. doi:10.1109/irps.2017.7936379

8. Lo, C.-L.; Catalano, M.; Smithe, K. K. H.; Wang, L.; Zhang, S.; Pop, E.; Kim, M. J.; Chen, Z. npj 2D Mater. Appl. 2017, 1, 42. doi:10.1038/s41699-017-0044-0

9. Lo, C.-L.; Zhang, K.; Smith, R. S.; Shah, K.; Robinson, J. A.; Chen, Z. IEEE Electron Device Lett. 2018, 39, 873-876. doi:10.1109/led.2018.2827061

10. Smithe, K. K. H.; Zhu, Z.; Bailey, C. S.; Pop, E.; Yoon, A. Investigation of monolayer MX2 as sub-nanometer copper diffusion barriers. In 2018 IEEE International Reliability Physics Symposium (IRPS), 2018; P-PI.1. doi:10.1109/irps.2018.8353681

11. Choi, W.; Choudhary, N.; Han, G. H.; Park, J.; Akinwande, D.; Lee, Y. H. Mater. Today 2017, 20, 116-130. doi:10.1016/j.mattod.2016.10.002

12. O'Dwyer, C.; Walsh, L. A.; Gity, F.; Bhattacharjee, S.; Hurley, P. K. Electrochem. Soc. Interface 2018, 27 (4), 53-58. doi:10.1149/2.f06184if

13. Zhang, Y.-H.; Chen, J.-L.; Yue, L.-J.; Zhang, H.-L.; Li, F. Comput. Theor. Chem. 2017, 1104, 12-17. doi:10.1016/j.comptc.2017.01.026
14. Mackin, C.; Fasoli, A.; Xue, M.; Lin, Y.; Adebiyi, A.; Bozano, L.; Palacios, T. 2D Mater. 2020, 7, 022002. doi:10.1088/2053-1583/ab6e88

15. Keong Koh, E. W.; Chiu, C. H.; Lim, Y. K.; Zhang, Y.-W.; Pan, H. Int. J. Hydrogen Energy 2012, 37, 14323-14328. doi:10.1016/j.ijhydene.2012.07.069

16. Zhang, C.; Jiao, Y.; Ma, F.; Kasi Matta, S.; Bottle, S.; Du, A. Beilstein J. Nanotechnol. 2018, 9, 1641-1646. doi:10.3762/bjnano.9.156

17. Lo, C.-L.; Helfrecht, B. A.; He, Y.; Guzman, D. M.; Onofrio, N.; Zhang, S.; Weinstein, D.; Strachan, A.; Chen, Z. J. Appl. Phys. 2020, 128, 080903. doi:10.1063/5.0013737

18. Ma, D.; Ju, W.; Li, T.; Zhang, X.; He, C.; Ma, B.; Tang, Y.; Lu, Z.; Yang, Z. Appl. Surf. Sci. 2016, 364, 181-189. doi:10.1016/j.apsusc.2015.12.142

19. Rawal, T. B.; Le, D.; Rahman, T. S. J. Phys. Chem. C 2017, 121 , 7282-7293. doi:10.1021/acs.jpcc.7b00036

20. Salehi, H.; Moaddeli, M.; Amiri, P. Surf. Sci. 2016, 647, 96-102. doi:10.1016/j.susc.2015.12.028

21. Wang, Y.; Wang, B.; Huang, R.; Gao, B.; Kong, F.; Zhang, Q. Phys. E (Amsterdam, Neth.) 2014, 63, 276-282. doi:10.1016/j.physe.2014.06.017

22. Zhao, R.; Lo, C.-L.; Zhang, F.; Ghosh, R. K.; Knobloch, T.; Terrones, M.; Chen, Z.; Robinson, J. Adv. Mater. Interfaces 2019, 6, 1901055. doi:10.1002/admi.201901055

23. Ersan, F.; Gökoğlu, G.; Aktürk, E. J. Phys. Chem. C 2015, 119 , 28648-28653. doi:10.1021/acs.jpcc.5b09034

24. Li, X. D.; Fang, Y. M.; Wu, S. Q.; Zhu, Z. Z. AIP Adv. 2015, 5, 057143. doi:10.1063/1.4921564

25. Makaremi, M.; Mortazavi, B.; Singh, C. V. J. Phys. Chem. C 2017, 121 18575-18583. doi:10.1021/acs.jpcc.7b04511

26. Smyth, C. M.; Addou, R.; McDonnell, S.; Hinkle, C. L.; Wallace, R. M. J. Phys. Chem. C 2016, 120, 14719-14729. doi:10.1021/acs.jpcc.6b04473

27. Fan, J.; Yang, J.; Pham, A.; Li, S. Phys. Chem. Chem. Phys. 2018, 20 18671-18677. doi:10.1039/c8cp02740d

28. Nies, C.-L.; Nolan, M. Beilstein J. Nanotechnol. 2020, 11, 391-406. doi:10.3762/bjnano.11.30

29. Addou, R.; Colombo, L.; Wallace, R. M. ACS Appl. Mater. Interfaces 2015, 7, 11921-11929. doi:10.1021/acsami.5b01778

30. Varela Pedreira, O.; Croes, K.; Leśniewska, A.; Wu, C.; van Der Veen, M. H.; de Messemaeker, J.; Vandersmissen, K.; Jourdan, N.; Wen, L. G.; Adelmann, C.; Briggs, B.; Gonzalez, V. V.; Bömmels, J.; Tőkei, Z. Reliability study on cobalt and ruthenium as alternative metals for advanced interconnects. In 2017 IEEE International Reliability Physics Symposium (IRPS), 2017; 6B-2. doi:10.1109/irps.2017.7936340

31. Bekiaris, N.; Wu, Z.; Ren, H.; Naik, M.; Park, J. H.; Lee, M.; Ha, T. H.; Hou, W.; Bakke, J. R.; Gage, M.; Wang, Y.; Tang, J. Cobalt fill for advanced interconnects. In 2017 IEEE International Interconnect Technology Conference (IITC), 2017; pp 1-3. doi:10.1109/iitc-amc.2017.7968981

32. Barmak, K.; Ezzat, S.; Gusley, R.; Jog, A.; Kerdsongpanya, S.; Khaniya, A.; Milosevic, E.; Richardson, W.; Sentosun, K.; Zangiabadi, A.; Gall, D.; Kaden, W. E.; Mucciolo, E. R.; Schelling, P. K.; West, A. C.; Coffey, K. R. J. Vac. Sci. Technol., A 2020, 38, 033406. doi:10.1116/6.0000018 
33. Pedreira, O. V.; Stucchi, M.; Gupta, A.; Gonzalez, V. V.; van der Veen, M.; Lariviere, S.; Wilson, C. J.; Tőkei, Z.; Croes, K. Metal reliability mechanisms in Ruthenium interconnects. In 2020 IEEE International Reliability Physics Symposium (IRPS), 2020; pp 1-7. doi:10.1109/irps45951.2020.9129087

34. Philip, T. M.; Lanzillo, N. A.; Gunst, T.; Markussen, T.; Cobb, J.; Aboud, S.; Robison, R. R. Phys. Rev. Appl. 2020, 13, 044045. doi:10.1103/physrevapplied.13.044045

35. Zhang, D.; Du, A.; Zhao, C.; Gao, J.; Mao, S.; Xu, J.; Gu, S.; Liu, Y.; Luo, X.; Li, Y.; Li, J.; Wang, W.; Chen, D.; Ye, T.; Luo, J. IEEE Trans. Electron Devices 2020, 67, 2076-2081. doi:10.1109/ted.2020.2983302

36. Zhang, J.; Xu, X.; Yang, L.; Cheng, D.; Cao, D. Small Methods 2019, 3, 1900653. doi:10.1002/smtd.201900653

37. Liu, J.; Zheng, Y.; Zhu, D.; Vasileff, A.; Ling, T.; Qiao, S.-Z. Nanoscale 2017, 9, 16616-16621. doi:10.1039/c7nr06111k

38. Kwon, I. S.; Debela, T. T.; Kwak, I. H.; Park, Y. C.; Seo, J.; Shim, J. Y.; Yoo, S. J.; Kim, J.-G.; Park, J.; Kang, H. S. Small 2020, 16, 2000081. doi:10.1002/smll.202000081

39. Young, B. T.; Pathan, M. A. K.; Jiang, T.; Le, D.; Marrow, N.; Nguyen, T.; Jordan, C. E.; Rahman, T. S.; Popolan-Vaida, D. M.; Vaida, M. E. J. Chem. Phys. 2020, 152, 074706. doi:10.1063/1.5129712

40. Liang, Z.; Shen, R.; Ng, Y. H.; Zhang, P.; Xiang, Q.; Li, X. J. Mater. Sci. Technol. 2020, 56, 89-121. doi:10.1016/j.jmst.2020.04.032

41. Rhatigan, S.; Michel, M.-C.; Nolan, M. JPhys Energy 2020, 2, 042002. doi:10.1088/2515-7655/aba3bc

42. Li, B.; Christiansen, C.; Badami, D.; Yang, C.-C. Microelectron. Reliab. 2014, 54, 712-724. doi:10.1016/j.microrel.2014.01.005

43. Edelstein, D. C. 20 Years of Cu BEOL in manufacturing, and its future prospects. In 2017 IEEE International Electron Devices Meeting (IEDM), 2017; 14.1. doi:10.1109/iedm.2017.8268387

44. Dong, C.; Li, Y.; Cheng, D.; Zhang, M.; Liu, J.; Wang, Y.-G.; Xiao, D.; Ma, D. ACS Catal. 2020, 10, 11011-11045.

doi:10.1021/acscatal.0c02818

45. Benia, H. M.; Lin, X.; Gao, H.-J.; Nilius, N.; Freund, H.-J. J. Phys. Chem. C 2007, 111, 10528-10533. doi:10.1021/jp0726891

46. Engel, J.; Francis, S.; Roldan, A. Phys. Chem. Chem. Phys. 2019, 21 , 19011-19025. doi:10.1039/c9cp03066b

47. Han, Y.; Ferrando, R.; Li, Z. Y. J. Phys. Chem. Lett. 2014, 5, 131-137. doi:10.1021/jz4022975

48. Kondati Natarajan, S.; Nies, C.-L.; Nolan, M. J. Chem. Phys. 2020, 152, 144701. doi:10.1063/5.0003852

49. Kresse, G.; Furthmüller, J. Phys. Rev. B 1996, 54, 11169-11186. doi:10.1103/physrevb.54.11169

50. Perdew, J. P.; Burke, K.; Ernzerhof, M. Phys. Rev. Lett. 1996, 77, 3865-3868. doi:10.1103/physrevlett.77.3865

51. Blöchl, P. E. Phys. Rev. B 1994, 50, 17953-17979. doi:10.1103/physrevb.50.17953

52. mp-54: Co (hexagonal, $\left.P 6_{3} / m m c, 194\right)$. https://materialsproject.org/materials/mp-54/ (accessed May 14, 2021). doi:10.17188/1263614

53. mp-2070: $\mathrm{CoS}_{2}$ (cubic, $\mathrm{Pa}-3,205$ ). https://materialsproject.org/materials/mp-2070/ (accessed May 14, 2021). doi:10.17188/1195854

54. mp-1139: $\mathrm{Co}_{3} \mathrm{Mo}$ (hexagonal, $P 6_{3} / m m c, 194$ ). https://materialsproject.org/materials/mp-1139/ (accessed May 14, 2021). doi:10.17188/1187793
55. mp-33: Ru (hexagonal, $\left.P 6_{3} / m m c, 194\right)$.

https://materialsproject.org/materials/mp-33/ (accessed May 14, 2021). doi:10.17188/1206459

56. mp-2030: RuS 2 (cubic, $\mathrm{Pa}-3,205$ ).

https://materialsproject.org/materials/mp-2030/ (accessed May 14, 2021). doi:10.17188/1195426

57. mp-975834: $\mathrm{Co}_{3} \mathrm{Mo}$ (hexagonal, $P 6_{3} / m m c, 194$ ). https://materialsproject.org/materials/mp-975834/ (accessed May 14, 2021). doi:10.17188/1314893

58. Kittel, C. Introduction to Solid State Physics; Wiley, 2004.

\section{License and Terms}

This is an Open Access article under the terms of the Creative Commons Attribution License (https://creativecommons.org/licenses/by/4.0). Please note that the reuse, redistribution and reproduction in particular requires that the author(s) and source are credited and that individual graphics may be subject to special legal provisions.

The license is subject to the Beilstein Journal of

Nanotechnology terms and conditions:

(https://www.beilstein-journals.org/bjnano/terms)

The definitive version of this article is the electronic one which can be found at: https://doi.org/10.3762/bjnano.12.56 